\title{
Polarization effects for pair creation by photon in oriented crystals at high energy
}

\author{
V. N. Baier and V. M. Katkov \\ Budker Institute of Nuclear Physics \\ 630090 Novosibirsk, Russia
}

November 6, 2018

\begin{abstract}
Pair creation by a photon in an oriented crystal can be considered in a frame of the quasiclassical operator method, which includes processes with polarized particles. Under some quite generic assumptions the general expression is derived for the probability of pair creation of longitudinally polarized electron (positron) by circularly polarized photon in oriented crystal. In particular cases $\vartheta_{0} \ll V_{0} / m$ and $\vartheta_{0} \gg V_{0} / m\left(\vartheta_{0}\right.$ is the angle of incidence, angle between the momentum of initial photon and axis (plane) of crystal, $V_{0}$ is the scale of a potential of axis or a plane relative to which the angle $\vartheta_{0}$ is defined) one has constant field approximation and the coherent pair production theory correspondingly. Side by side with coherent process the probability of incoherent pair creation is calculated, which differs essentially from amorphous one. At high energy the pair creation in oriented crystals is strongly enhanced comparing with amorphous medium. In appendixes the integral polarization of positron is found in external field and for coherent and incoherent mechanisms.
\end{abstract}




\section{Introduction}

The study of processes with participation of polarized electrons and photons permits to obtain the important physical information. Because of this reason the experiments with use of polarized particles are performed and are planning in many laboratories (CERN, Jefferson Nat Accl Fac, SLAC, BINP, etc). In this paper it is shown that oriented crystal is an unique tool for work with polarized electrons and photons

The quasiclassical operator method developed by authors [1]-3 is adequate for consideration of the electromagnetic processes at high energy. The probability of polarized pair creation by a circularly polarized photon has a form (see [4], p.73, Eq.(3.12); the method is given also in [5], [6])

$$
d w=\frac{e^{2}}{(2 \pi)^{2}} \frac{d^{3} p}{\omega} \int d t_{2} \int d t_{1} R_{s \bar{s}}^{(\lambda)}\left(t_{2}\right) R_{s \bar{s}}^{(\lambda) *}\left(t_{1}\right) \exp \left[\frac{i \varepsilon}{\varepsilon^{\prime}}\left(k x\left(t_{2}\right)-k x\left(t_{1}\right)\right)\right],
$$

where for polarized electrons and positrons one has within the relativistic accuracy

$$
R_{s \bar{s}}^{(\lambda)}=\frac{m}{\sqrt{\varepsilon \varepsilon^{\prime}}} u_{s}^{+}(p) \mathbf{e}^{\lambda} \boldsymbol{\alpha} v_{\bar{s}}\left(p^{\prime}\right)
$$

here $k^{\nu}=(\omega, \mathbf{k})$ is the 4 -momentum of the initial photon, $p^{\mu}=(\varepsilon, \mathbf{p})$ is the 4-momentum of the created electron, $k^{2}=0, x^{\mu}(t)=(t, \mathbf{r}(t)), t$ is the time, and $\mathbf{r}(t)$ is the created electron location on a classical trajectory, $k x(t)=\omega t-\mathbf{k r}(t)$, $\varepsilon^{\prime}=\omega-\varepsilon, \mathbf{p}^{\prime}=\mathbf{k}-\mathbf{p}, \lambda= \pm 1$ is the circular polarization of photon, $s(\bar{s})= \pm 1$ is the longitudinal(with respect to direction of motion) polarization of electron (positron), we employ units such that $\hbar=c=1$.

Passing on to two-component spinors $\varphi$ and choosing as the quantization axis (the axis $z$ ) the direction of photon momentum $\mathbf{k}$ we get

$$
\begin{aligned}
& \mathbf{e}^{(\lambda)}=\frac{1}{\sqrt{2}}\left(\mathbf{e}_{x}+i \lambda \mathbf{e}_{y}\right), \quad \mathbf{e}^{\lambda} \boldsymbol{\alpha}=\sqrt{2}\left(\begin{array}{cc}
0 & \hat{s}_{\lambda} \\
\hat{s}_{\lambda} & 0
\end{array}\right), \\
& \sqrt{\frac{m}{\varepsilon}} u_{s}(p)=\sqrt{\frac{\varepsilon+m}{2 \varepsilon}}\left(\begin{array}{c}
\varphi_{s} \\
\frac{\boldsymbol{\sigma} \mathbf{p}}{\varepsilon+m} \varphi_{s}
\end{array}\right), \\
& \sqrt{\frac{m}{\varepsilon^{\prime}}} v_{\bar{s}}\left(p^{\prime}\right)=\sqrt{\frac{\varepsilon^{\prime}+m}{2 \varepsilon^{\prime}}}\left(\begin{array}{c}
\frac{\boldsymbol{\sigma} \mathbf{p}^{\prime}}{\varepsilon^{\prime}+m} \varphi_{-\bar{s}} \\
\varphi_{-\bar{s}}
\end{array}\right) .
\end{aligned}
$$

Here $\hat{s}_{ \pm}=\left(\sigma_{x} \pm i \sigma_{y}\right) / 2$ is the raising (lowering) operator:

$$
\sigma_{z} \varphi_{s}=s \varphi_{s}, \quad \hat{s}_{\lambda} \varphi_{s}=\delta_{\lambda,-s} \varphi_{-s}, \quad \varphi_{s}^{+} \hat{s}_{\lambda}=\delta_{\lambda, s} \varphi_{-s}^{+}
$$

Below we will consider the small angle approximation (the vector $\mathbf{p}$ is directed nearly along the vector $\mathbf{k}$, just this configuration yields the main contribution into 
the process probability) and will retain only the main terms in the decomposition over $1 / \gamma(\gamma=\varepsilon / m$ is the Lorentz factor $)$. Within this accuracy one obtains

$$
\begin{aligned}
& \sqrt{\frac{m}{\varepsilon}} u_{s}(p)=\frac{1}{\sqrt{2}}\left(\left[s\left(1-\frac{m}{\varepsilon}\right)+\boldsymbol{\sigma} \boldsymbol{\vartheta}\right] \varphi_{s}\right) \\
& \sqrt{\frac{m}{\varepsilon^{\prime}}} v_{\bar{s}}\left(p^{\prime}\right)=\frac{1}{\sqrt{2}}\left(\begin{array}{c}
-\left[\bar{s}\left(1-\frac{m}{\varepsilon^{\prime}}\right)+\frac{\varepsilon}{\varepsilon^{\prime}} \boldsymbol{\sigma} \boldsymbol{\vartheta}\right] \varphi_{-\bar{s}} \\
\varphi_{-\bar{s}}
\end{array}\right),
\end{aligned}
$$

where $\boldsymbol{\vartheta}=\mathbf{v}-\mathbf{n}=\mathbf{v}_{\perp}, \mathbf{v}=\mathbf{v}(t)$ is the electron velocity, $\mathbf{v}_{\perp}$ is the component of electron velocity perpendicular to the vector $\mathbf{n}=\mathbf{k} / \omega$. Let us note that $\boldsymbol{\vartheta}^{\prime}=\mathbf{v}^{\prime}-\mathbf{n}=\mathbf{p}^{\prime} / \varepsilon^{\prime}-\mathbf{n}=(\mathbf{n}-\mathbf{v}) \varepsilon / \varepsilon^{\prime}, \vartheta^{\prime} \gamma^{\prime}=\vartheta \gamma$.

Using the relations

$$
\begin{aligned}
& \varphi_{s}^{+} \boldsymbol{\sigma} \boldsymbol{\vartheta} \varphi_{s}=0, \quad \varphi_{-s}^{+} \boldsymbol{\sigma} \boldsymbol{\vartheta} \varphi_{s}=\vartheta_{x}+i s \vartheta_{y} \equiv \vartheta^{(s)}, \quad \varphi_{s}^{+} \hat{s}_{\lambda} \varphi_{-\bar{s}}=\delta_{\lambda, s} \delta_{\lambda, \bar{s}}, \\
& \varphi_{s}^{+} \hat{s}_{\lambda} \boldsymbol{\sigma} \boldsymbol{\vartheta} \varphi_{-\bar{s}}=\delta_{\lambda, s} \varphi_{-s}^{+} \boldsymbol{\sigma} \boldsymbol{\vartheta} \varphi_{-\bar{s}}=\vartheta^{(\lambda)} \delta_{\lambda, s} \delta_{\lambda,-\bar{s}}, \\
& \varphi_{s}^{+} \boldsymbol{\sigma} \boldsymbol{\vartheta} \hat{s}_{\lambda} \varphi_{-\bar{s}}=\delta_{\lambda, \bar{s}} \varphi_{s}^{+} \boldsymbol{\sigma} \boldsymbol{\vartheta} \varphi_{\bar{s}}=\vartheta^{(\lambda)} \delta_{\lambda, \bar{s}} \delta_{\lambda,-s},
\end{aligned}
$$

we find within the adopted accuracy

$$
R_{s \bar{s}}^{(\lambda)}=\frac{1}{\sqrt{2}}\left[\frac{m \omega}{\varepsilon \varepsilon^{\prime}} \delta_{\lambda, s} \delta_{\lambda, \bar{s}}-\vartheta^{(\lambda)}\left(\delta_{\lambda, \bar{s}} \delta_{\lambda,-s}+\frac{\varepsilon}{\varepsilon^{\prime}} \delta_{\lambda, s} \delta_{\lambda,-\bar{s}}\right)\right] .
$$

Taking advantage of relation $\delta_{\lambda, s}^{2}=\delta_{\lambda, s}=(1+\xi) / 2$, where $\xi=\lambda s$, we obtain

$$
\begin{aligned}
& R_{s \bar{s}}^{(\lambda)}\left(t_{2}\right) R_{s \bar{s}}^{(\lambda) *}\left(t_{1}\right)=\frac{m^{2}}{8 \varepsilon_{-}^{2} \varepsilon_{+}^{2}}\left\{\omega^{2}\left(1+\xi_{-}\right)\left(1+\xi_{+}\right)\right. \\
& \left.+\gamma^{2} \vartheta_{2}^{(\lambda)} \vartheta_{1}^{(\lambda) *}\left[\varepsilon_{+}^{2}\left(1-\xi_{-}\right)\left(1+\xi_{+}\right)+\varepsilon_{-}^{2}\left(1+\xi_{-}\right)\left(1-\xi_{+}\right)\right]\right\} .
\end{aligned}
$$

Here for visualization we have written $\varepsilon=\varepsilon_{-}$for the electron energy, $\varepsilon^{\prime}=\varepsilon_{+}$for the positron energy, $\xi_{-}=\lambda s, \xi_{+}=\lambda \bar{s}$ for description of electron and positron polarization correspondingly, $\vartheta_{2,1} \equiv \vartheta\left(t_{2,1}\right)$,

$$
\vartheta_{2}^{(\lambda)} \vartheta_{1}^{(\lambda) *}=\left(\vartheta_{x}\left(t_{2}\right)+i \lambda \vartheta_{y}\left(t_{2}\right)\right)\left(\vartheta_{x}\left(t_{1}\right)-i \lambda \vartheta_{y}\left(t_{1}\right)\right)=\boldsymbol{\vartheta}_{2} \boldsymbol{\vartheta}_{1}-i \lambda\left(\mathbf{n}\left(\boldsymbol{\vartheta}_{2} \times \boldsymbol{\vartheta}_{1}\right)\right) .
$$

Substituting Eqs.(1.8), (1.9) into Eq.(1.1) one obtains the completely differential probability of pair creation for circularly polarized photon and longitudinally polarized electron and positron. The above analysis shows that in the situation under consideration the derivation with direct calculation of matrix element is very simple comparing with the standard procedure (with calculation of traces for polarized particles). This is in consequence of Eq.(1.6).

It follows from Eqs.(1.7), (1.8) that in the case when the energy of one of particles is essentially larger than other particle energy, then together with energy the polarization of photon is transmitted to this particle. This conclusion can be 
obtained immediately from Eqs.(1.3), (1.4) if one neglects the angle $\boldsymbol{\vartheta}$ in the wave function Eq. (1.5) for the particle with large energy and reserves it in the wave function for the particle with small energy only. Then either one wave function or another becomes proportional to either $\varphi_{s}$ or $\varphi_{-\bar{s}}$. In this case the polarization of particle (see Eq.(1.4) is defined uniquely. If in this case in addition the angles $\vartheta \gg 1 / \gamma$ contribute mainly $(\vartheta$ is the angle between momenta of initial photon and created particle), then one can neglect the terms in Eqs.(1.7), (11.8) with the same polarizations of created particles. Then in Eq.(1.8) only one term remains which defines uniquely the polarizations of both particles. This fact agrees with helicity conservation rule in electromagnetic processes. This situation is realized in the process of pair creation by a photon in an external field for $\kappa \gg 1$ [4], as well as for the same process under strong influence of the LPM effect [1].

Summing in Eq.(1.8) over the polarizations of created electron $\xi_{-}$and omitting the term $\left(\mathbf{v}\left(\boldsymbol{\vartheta}_{1} \times \boldsymbol{\vartheta}_{2}\right)\right)$ which vanishes at integration over the azimuthal angle of created pair we get

$$
\sum_{\xi_{-}} R_{s \bar{s}}^{(\lambda)}\left(t_{2}\right) R_{s \bar{s}}^{(\lambda) *}\left(t_{1}\right)=\frac{m^{2}}{8 \varepsilon_{-}^{2} \varepsilon_{+}^{2}}\left\{\omega^{2}\left(1+\xi_{+}\right)+\gamma^{2} \boldsymbol{\vartheta}_{2} \boldsymbol{\vartheta}_{1}\left[\varepsilon_{+}^{2}\left(1+\xi_{+}\right)+\varepsilon_{-}^{2}\left(1-\xi_{+}\right)\right]\right\} .
$$

The cross process of emission of photon with energy $\omega$ by an electron with high energy $\varepsilon$ in oriented crystal was considered recently by authors [12. It is evident that this process possesses the similar properties in the case when the photon takes away an essential part of electron energy $\left(\varepsilon^{\prime}=\varepsilon-\omega \ll \varepsilon\right)$. The explicit expression for the transformed combination $R_{s \bar{s}}^{(\lambda)}\left(t_{2}\right) R_{s \bar{s}}^{(\lambda) *}\left(t_{1}\right)$ in Eq.(1.8) can be found using the standard substitutions:

$$
\begin{aligned}
& \varepsilon_{-} \rightarrow \varepsilon^{\prime}, \quad \varepsilon_{+} \rightarrow-\varepsilon, \quad \omega \rightarrow-\omega, \quad \lambda \rightarrow-\lambda, \\
& \bar{s} \rightarrow-\zeta, \quad s \rightarrow \zeta^{\prime}, \quad \xi_{+} \rightarrow \xi, \quad \xi_{-} \rightarrow-\xi^{\prime} .
\end{aligned}
$$

As a result we find

$$
\begin{aligned}
& R_{\zeta^{\prime} \zeta}^{(\lambda) *}\left(t_{2}\right) R_{\zeta^{\prime} \zeta}^{(\lambda)}\left(t_{1}\right)=\frac{m^{2}}{8 \varepsilon^{\prime 2} \varepsilon^{2}}\left\{\omega^{2}(1+\xi)\left(1-\xi^{\prime}\right)\right. \\
& +\gamma^{2}\left(\boldsymbol{\vartheta}_{1} \boldsymbol{\vartheta}_{2}+i \lambda\left(\mathbf{v}\left(\boldsymbol{\vartheta}_{1} \times \boldsymbol{\vartheta}_{2}\right)\right)\left[\varepsilon^{2}(1+\xi)\left(1+\xi^{\prime}\right)+\varepsilon^{\prime 2}(1-\xi)\left(1-\xi^{\prime}\right)\right]\right\}
\end{aligned}
$$

where $\xi=\lambda \zeta, \xi^{\prime}=\lambda \zeta^{\prime}$. Substituting Eq.(1.12) into the formula for the probability of photon emission (see e.g. [4], p.63, Eq.(2.27)) one obtains the completely differential probability of radiation of circularly polarized photon from longitudinally polarized electron in the case when the final electron has longitudinal polarization Summing in Eq.(1.12) over polarizations of final electron $\zeta^{\prime}$ and omitting the term $\left(\mathbf{v}\left(\boldsymbol{\vartheta}_{1} \times \boldsymbol{\vartheta}_{2}\right)\right)$ which vanishes at integration over angles of emitted photon we arrive to Eq.(1.8) of [12. 
It should be noted that while the terms depending on angles in Eqs. (1.8) and (1.12) describe the spin correlations arising from helicity conservation rule, the spin correlations in terms $\propto \omega^{2}$ describes by rule of conservation of projection of angular momentum on the direction of motion at zero angles. This explains absence of the term with $\left(1-\xi_{-}\right)\left(1-\xi_{+}\right)$in Eq.(1.8) and the term with $(1-$ $\xi)\left(1+\xi^{\prime}\right)$ in Eq.(1.12).

It should be noted that a few different spin correlations are known in an external field. But after averaging over directions of crystal field only the longitudinal polarization considered here survives.

\section{General approach to pair creation in oriented crystal}

The theory of high-energy electron radiation and electron-positron pair creation in oriented crystals was developed in [7]- 8], and given in [4]. In these publications the process radiation from electron and pair creation by a photon was considered for unpolarized particles. Since the expression for the pair creation probability has the same structure as Eqs.(1.8), (1.10) below we use systematically the methods of mentioned papers to obtain the characteristics of pair creation of longitudinally polarized electron (positron) by a circularly polarized photon.

Let $\vartheta_{0}$ be the photon angle of incidence with respect of a chosen axis of crystal and $V_{0}$ be the scale of the corresponding continuous potential of the axis. For $\vartheta_{0} \gg\left(V_{0} / \varepsilon\right)^{1 / 2} \equiv \vartheta_{c}$, created particles are moving high above the potential barrier, and can be described in terms of rectilinear trajectory. In rest frame of a crystal there is only an electric field of axis $\mathbf{E}$. In frame moving with a relativistic velocity $\mathbf{v}=\mathbf{n} v\left(\mathbf{n}=\mathbf{k} / \omega, k^{2}=\omega^{2}-\mathbf{k}^{2}=0\right)$ along the photon direction momentum, a magnetic field $\mathbf{H}=\gamma_{v}(\mathbf{E} \times \mathbf{v})$ arises $\left(\gamma_{v}=\left(1-v^{2}\right)^{-1 / 2} \gg 1\right)$ and, as it is well known, that resultant field in this frame can be represented with relativistic accuracy in the form of plane waves with frequencies $\gamma_{v}\left|q_{\|}\right| v\left(q_{\|}=\mathbf{q n}\right)$.

The periodic crystal potential $U(\mathbf{r})$ can be presented as the Fourier series (see e.g. 4], Sec.8 )

$$
U(\mathbf{r})=\sum_{\mathbf{q}} G(\mathbf{q}) e^{-i \mathbf{q} \mathbf{r}},
$$

where $\mathbf{q}=2 \pi\left(n_{1}, n_{2}, n_{3}\right) / l ; l$ is the lattice constant.

The equivalent photon flux averaged over time and over transverse coordinates is the sum of partial contributions $\mathbf{J}_{q}$. The later quantity has the form

$$
\mathbf{J}_{q}=-\mathbf{n} \frac{\gamma_{v}}{4 \pi e^{2}} \frac{|G(\mathbf{q})|^{2}}{\left|q_{\|}\right|} \mathbf{q}_{\perp}^{2}
$$

where $\mathbf{q}_{\perp}=\mathbf{q}-\mathbf{n}(\mathbf{q n})$. In the interaction region, the transverse size of which is of the order $\lambda_{c}=1 / m$, and the longitudinal size is the process formation 
length, which is of the order $2 \pi / \gamma_{v} q_{\|}$in the center mass frame of the incident and equivalent photons, there are $N_{q} \simeq 2 \pi \lambda_{c}^{2}\left|\mathbf{J}_{q} / \gamma_{v} q_{\|}\right|$photons. The effective strength of interaction is characterized by the parameter

$$
\alpha N_{p h}=\alpha \sum_{\mathbf{q}} N_{\mathbf{q}}=\sum_{\mathbf{q}} \frac{|G(\mathbf{q})|^{2}}{m^{2}\left|q_{\|}\right|^{2}} \mathbf{q}_{\perp}^{2}
$$

This parameter is purely classical (it does not contain Planck's constant $\hbar$ ) and always arises in problems with electromagnetic interaction in external field. For $\alpha N_{p h} \ll 1$ the external field can be taken into account in perturbation theory, while for $\alpha N_{p h} \gg 1$ one has the constant field limit as it is known from the theory of interaction of photon with the plane wave field (see e.g. [9]). For estimates one can assume $|G(\mathbf{q})| \sim V_{0}, q_{\|} \sim q_{\perp} \vartheta_{0}$, in which case

$$
\alpha N_{p h} \sim\left(\frac{V_{0}}{m \vartheta_{0}}\right)^{2}
$$

Therefore for $\vartheta_{0} \ll V_{0} / m \equiv \vartheta_{v}$ the constant field approximation is applicable while for $\vartheta_{0} \gg V_{0} / m$ the perturbation theory is valid, the first approximation of which is the coherent pair production theory (see e.g. [10]).

In a crystal one have to integrate over pair creation points. Substituting Eq.(1.10) into Eq.(1.1) we find for the probability of creation of longitudinally polarized positron by a circularly polarized photon

$$
\begin{aligned}
& d w_{\xi_{+}}=\frac{\alpha m^{2}}{(2 \pi)^{2} \omega} \frac{d^{3} p_{+}}{2 \varepsilon_{-} \varepsilon_{+}} \int \frac{d^{3} r_{0}}{V} \\
& \times \int d t_{1} \int d t_{2} e^{i A_{p}}\left[\varphi_{p 1}\left(\xi_{+}\right)-\frac{1}{4} \gamma^{2}\left(\mathbf{v}_{1}-\mathbf{v}_{2}\right)^{2} \varphi_{p 2}\left(\xi_{+}\right)\right], \\
& A_{p}=\frac{m^{2} \omega}{2 \varepsilon_{+} \varepsilon_{-}} \int_{t_{1}}^{t_{2}}\left[1+\gamma^{2} \boldsymbol{\vartheta}^{2}(t)\right] d t \\
& \varphi_{p 1}\left(\xi_{+}\right)=1+\xi_{+} \frac{\omega}{\varepsilon_{+}}, \quad \varphi_{p 2}\left(\xi_{+}\right)=\left(1+\xi_{+}\right) \frac{\varepsilon_{+}}{\varepsilon_{-}}+\left(1-\xi_{+}\right) \frac{\varepsilon_{-}}{\varepsilon_{+}},
\end{aligned}
$$

where $\alpha=e^{2}=1 / 137$, the vector $\boldsymbol{\vartheta}$ is defined in Eq.(1.5), $V$ is the volume of the crystal, $\mathbf{p}_{+}$is the momentum of the positron in the creation point $\mathbf{r}_{0}$. Corresponding, the probability of creation of longitudinally polarized electron by a circularly polarized photon is

$$
\begin{aligned}
& d w_{\xi_{-}}=\frac{\alpha m^{2}}{(2 \pi)^{2} \omega} \frac{d^{3} p_{-}}{2 \varepsilon_{-} \varepsilon_{+}} \int \frac{d^{3} r_{0}}{V} \\
& \times \int d t_{1} \int d t_{2} e^{i A_{p}}\left[\varphi_{e 1}\left(\xi_{-}\right)-\frac{1}{4} \gamma^{2}\left(\mathbf{v}_{1}-\mathbf{v}_{2}\right)^{2} \varphi_{e 2}\left(\xi_{-}\right)\right], \\
& \varphi_{e 1}\left(\xi_{-}\right)=1+\xi_{-} \frac{\omega}{\varepsilon_{-}}, \quad \varphi_{e 2}\left(\xi_{-}\right)=\left(1-\xi_{-}\right) \frac{\varepsilon_{+}}{\varepsilon_{-}}+\left(1+\xi_{-}\right) \frac{\varepsilon_{-}}{\varepsilon_{+}} .
\end{aligned}
$$


From Eq.(2.5) one can find polarization of created positron

$$
\boldsymbol{\zeta}_{+}=\zeta_{p} \lambda \mathbf{v}, \quad \zeta_{p}=\frac{d w_{\xi_{+}=1}-d w_{\xi_{+}=-1}}{d w_{\xi_{+}=1}+d w_{\xi_{+}=-1}}
$$

In the limiting case $\varepsilon_{+} \gg \varepsilon_{-}$it follows from Eq.(2.7) that $\zeta_{p} \rightarrow 1$.

The particle velocity can be presented in a form $\mathbf{v}(t)=\mathbf{v}_{0}+\Delta \mathbf{v}(t)$, where $\mathbf{v}_{0}$ is the average velocity. If $\vartheta_{0} \gg \vartheta_{c}$, we find $\Delta \mathbf{v}(t)$ using the rectilinear trajectory approximation for the potential Eq.(2.1)

$$
\Delta \mathbf{v}(t)=-\frac{1}{\varepsilon} \sum \frac{G(\mathbf{q})}{q_{\|}} \mathbf{q}_{\perp} \exp \left[-i\left(q_{\|} t+\mathbf{q r}\right)\right],
$$

where $q_{\|}=(\mathbf{q n}), \mathbf{q}_{\perp}=\mathbf{q}-\mathbf{n}(\mathbf{q n})$ (for detail see Eqs.(3.27)-(3.31) in [4]). Substituting Eq.(2.8) into Eq.(2.5) and performing integration over $\mathbf{u}=\mathbf{n}-\mathbf{v}_{0}\left(d^{3} p \simeq\right.$ $\varepsilon^{2} d \varepsilon d \mathbf{u}$ ) and passing to the variables $t, \tau: t_{1}=t-\tau, t_{2}=t+\tau$, we obtain after simple calculations the general expression for the probability of creation of longitudinally polarized positron by a circularly polarized photon

$$
\begin{aligned}
& d W_{\xi_{+}} \equiv \frac{d w_{\xi_{+}}}{d t}=\frac{i \alpha m^{2}}{4 \pi \omega^{2}} d \varepsilon_{+} \int \frac{d^{3} r_{0}}{V} \int \frac{d \tau}{\tau+i 0}\left[\varphi_{p 1}\left(\xi_{+}\right)+\varphi_{p 2}\left(\xi_{+}\right)\right. \\
& \left.\times\left(\sum_{\mathbf{q}} \frac{G(\mathbf{q})}{m q_{\|}} \mathbf{q}_{\perp} \sin \left(q_{\|} \tau\right) e^{i \mathbf{q} \mathbf{r}_{0}}\right)^{2}\right] e^{i A_{p 1}},
\end{aligned}
$$

where

$$
\begin{aligned}
& A_{p 1}=\frac{m^{2} \omega \tau}{\varepsilon_{-} \varepsilon_{+}}\left[1+\sum_{\mathbf{q}, \mathbf{q}^{\prime}} \frac{G(\mathbf{q}) G\left(\mathbf{q}^{\prime}\right)}{m^{2} q_{\|} q_{\|}^{\prime}}\left(\mathbf{q}_{\perp} \mathbf{q}_{\perp}^{\prime}\right) \Psi\left(q_{\|}, q_{\|}^{\prime}, \tau\right) \exp \left[-i\left(\mathbf{q}+\mathbf{q}^{\prime}\right) \mathbf{r}_{0}\right]\right] \\
& \Psi\left(q_{\|}, q_{\|}^{\prime}, \tau\right)=\frac{\sin \left(q_{\|}+q_{\|}^{\prime}\right) \tau}{\left(q_{\|}+q_{\|}^{\prime}\right) \tau}-\frac{\sin \left(q_{\|} \tau\right)}{q_{\|} \tau} \frac{\sin \left(q_{\|}^{\prime} \tau\right)}{q_{\|}^{\prime} \tau}
\end{aligned}
$$

\section{Pair creation for $\vartheta_{0} \ll V_{0} / m$ (constant field limit and corrections to it)}

The behavior of probability Eq.(2.9) for various entry angles and energies is determined by the dependence on these parameters of the phase $A_{p 1}$ given Eq.(2.10). Here we consider the axial case for $\vartheta_{0} \ll V_{0} / m \equiv \vartheta_{v}$. The direction of crystal axis we take as $z$-axis of the coordinate system. The order of magnitude of the double sum in $A_{p 1}$ is $(G / m)^{2}\left(q_{\perp} / q_{\|}\right)^{2} \Psi\left(q_{\|}, q_{\|}^{\prime}, \tau\right)$. For the vector q lying in the plane $(x, y)$ we introduce notation $\mathbf{q}_{t}$, for such vectors one has $q_{z}=0$ and the quantities in Eq.(2.10) can be estimated in the following way:

$$
G(\mathbf{q}) \sim V_{0}, \quad q_{\perp} \sim 1 / a, \quad q_{\|} \sim \vartheta_{0} / a,
$$


where $a$ is the size of the region of action of the continuous potential. For all remaining vectors $q_{\perp} \sim q_{\|} \sim 1 / a$. Then the contribution to the sum of the terms with $q_{z} \neq 0$ will be $\sim\left(V_{0} / m\right)^{2} \Psi \leq\left(V_{0} / m\right)^{2}$. Since $\left(V_{0} / m\right)^{2} \ll 1$ this contribution can be neglected. Thus, we keep in the sum only terms with $\mathbf{q}_{t}$ for which its value is $\sim\left(V_{0} / m \vartheta_{0}\right)^{2} \Psi$. The large value of the phase $A_{p 1}$ leads to an exponential suppression of probability $d W_{\xi_{+}}$. Therefore the characteristic value of the variable $\tau$ in the integral Eq.(2.10) (which have the meaning of the formation time (length) of the process) will be adjusted in a such way that the large factor $\left(V_{0} / m \vartheta_{0}\right)^{2}$ will be compensated by the function $\Psi\left(q_{\|}, q_{\|}^{\prime}, \tau\right)$, i.e. for small entry angles the contribution gives region where $q_{\|} \tau \ll 1$. Expanding the phase $A_{p 1}$ correspondingly we find an approximate expression for $\vartheta_{0} \ll \vartheta_{v}$

$$
\begin{aligned}
& A_{p 1} \simeq \frac{m^{2} \omega \tau}{\varepsilon_{-} \varepsilon_{+}}\left\{1-\frac{\tau^{2}}{3} \sum_{\mathbf{q}_{t}, \mathbf{q}_{t}^{\prime}} G\left(\mathbf{q}_{t}\right) G\left(\mathbf{q}_{t}^{\prime}\right) \frac{\left(\mathbf{q}_{t} \mathbf{q}_{t}^{\prime}\right)}{m^{2}} \exp \left[-i\left(\mathbf{q}_{t}+\mathbf{q}_{t}^{\prime}\right) \boldsymbol{\varrho}\right]\right. \\
& \left.\times\left[1-\frac{\tau^{2}}{10}\left(\left(\mathbf{n} \mathbf{q}_{t}\right)^{2}+\left(\mathbf{n} \mathbf{q}_{t}^{\prime}\right)^{2}+\frac{2}{3}\left(\mathbf{n} \mathbf{q}_{t}\right)\left(\mathbf{n} \mathbf{q}_{t}^{\prime}\right)\right)\right]\right\}
\end{aligned}
$$

here $\varrho=\mathbf{r}_{0 t}$. We can rewrite Eq.(3.2) in the terms of the average potential of atomic string $U(\boldsymbol{\varrho})=\sum_{\mathbf{q}_{t}} G\left(\mathbf{q}_{t}\right) \exp \left(-i \mathbf{q}_{t} \boldsymbol{\varrho}\right)$ :

$$
A_{p 1}=\frac{m^{2} \omega \tau}{\varepsilon_{-} \varepsilon_{+}}\left\{1+\frac{\tau^{2}}{3} \mathbf{b}^{2} \tau^{2}+\frac{\tau^{4}}{15}\left[\left(\mathbf{b}(\mathbf{n} \boldsymbol{\nabla})^{2} \mathbf{b}\right)+\frac{1}{3}((\mathbf{n} \boldsymbol{\nabla}) \mathbf{b})^{2}\right]\right\},
$$

where $\mathbf{b}=\boldsymbol{\nabla} U(\boldsymbol{\varrho}) / m, \boldsymbol{\nabla}=\partial / \partial \boldsymbol{\varrho}$. For the pre-exponential factor in Eq.(2.9) we find

$$
[\ldots] \simeq \varphi_{p 1}\left(\xi_{+}\right)-\varphi_{p 2}\left(\xi_{+}\right) \tau^{2}\left[\mathbf{b}^{2}+\frac{\tau^{2}}{3}(\mathbf{b}(\mathbf{n} \nabla) \mathbf{b})\right]
$$

Taking the integral over $\tau$ we obtain the spectral probability for $\vartheta_{0} \ll V_{0} / m$.

$$
\begin{aligned}
& d W_{\xi_{+}}^{F}\left(\varepsilon_{+}\right)=\frac{\alpha m^{2} d \varepsilon_{+}}{2 \sqrt{3} \pi \omega^{2}} \int \frac{d^{2} \varrho}{S}\left\{R_{0}(\lambda)-\frac{\left(\mathbf{b}(\mathbf{n} \boldsymbol{\nabla})^{2} \mathbf{b}\right)}{3 \mathbf{b}^{4}} R_{1}(\lambda)\right. \\
& \left.-\frac{\lambda}{30 \mathbf{b}^{4}}\left[((\mathbf{n} \boldsymbol{\nabla}) \mathbf{b})^{2}+3\left(\mathbf{b}(\mathbf{n} \boldsymbol{\nabla})^{2} \mathbf{b}\right)\right] R_{2}(\lambda)\right\},
\end{aligned}
$$

where

$$
\begin{aligned}
& R_{0}(\lambda)=\varphi_{p 2}\left(\xi_{+}\right) K_{2 / 3}(\lambda)+\varphi_{p 1}\left(\xi_{+}\right) \int_{\lambda}^{\infty} K_{1 / 3}(y) d y \\
& R_{1}(\lambda)=\varphi_{p 2}\left(\xi_{+}\right)\left(K_{2 / 3}(\lambda)-\frac{2}{3 \lambda} K_{1 / 3}(\lambda)\right) \\
& R_{2}(\lambda)=\varphi_{p 1}\left(\xi_{+}\right)\left(K_{1 / 3}(\lambda)-\frac{4}{3 \lambda} K_{2 / 3}(\lambda)\right) \\
& -\varphi_{p 2}\left(\xi_{+}\right)\left(\frac{4}{\lambda} K_{2 / 3}(\lambda)-\left(1+\frac{16}{9 \lambda^{2}}\right) K_{1 / 3}(\lambda)\right)
\end{aligned}
$$


here $\lambda=\frac{2 m^{2} \omega}{3 \varepsilon_{-} \varepsilon_{+}|\mathbf{b}|}, K_{\nu}(\lambda)$ is the modified Bessel function (McDonald's function). Since the expression for $d W_{\xi_{+}}^{F}$ is independent of $z$, it follows that $\int d^{3} r_{0} / V \rightarrow$ $\int d^{2} \varrho / s$, where $S$ is the transverse cross section area per axis. The term in Eq.(3.5) with $R_{0}(\lambda)$ represent the spectral probability in the constant field limit. The other terms are the correction proportional $\vartheta_{0}^{2}$ arising due to nongomogeneity of field in crystal.

If the potential $U(\varrho)$ can be assumed to be axially symmetric, we put $U=$ $U\left(\boldsymbol{\varrho}^{2}\right)$ and one can integrate over angles of vector $\varrho$. We obtain

$$
\begin{aligned}
& d W_{\xi_{+}}^{F}\left(\varepsilon_{+}\right)=\frac{\alpha m^{2} d \varepsilon_{+}}{2 \sqrt{3} \pi \omega^{2}} \int_{0}^{x_{0}} \frac{d x}{x_{0}}\left\{R_{0}(\lambda)-\frac{1}{6}\left(\frac{m \vartheta_{0}}{V_{0}}\right)^{2}\left[\frac{x g^{\prime \prime}+2 g^{\prime}}{x g^{3}} R_{1}(\lambda)\right.\right. \\
& \left.\left.-\frac{\lambda}{20 g^{4} x^{2}}\left(2 x^{2} g^{\prime 2}+g^{2}+14 g g^{\prime} x+6 x^{2} g g^{\prime \prime}\right) R_{2}(\lambda)\right]\right\}
\end{aligned}
$$

where we have gone over to the new variable $x=\varrho^{2} / a_{s}^{2}, x \leq x_{0}, x_{0}^{-1}=\pi a_{s}^{2} d n_{a}=$ $\pi a_{s}^{2} / S, a_{s}$ is the effective screening radius of the potential of the string, $n_{a}$ is the density of atoms in a crystal, $d$ is the average distance between atoms of a chain forming the axis. The notation $U^{\prime}(x)=-V_{0} g(x)$ is used in Eq.(3.7) and

$$
\lambda=\frac{2 \omega^{2}}{3 \varepsilon_{-} \varepsilon_{+} \kappa(x)}, \kappa_{s}=\frac{V_{0} \omega}{m^{3} a_{s}}, \kappa(x)=-\frac{d U}{d \varrho} \frac{\omega}{m^{3}}=2 \kappa_{s} \sqrt{x} g(x) .
$$

For specific calculation we use the following expression for the potential of axis:

$$
U(x)=V_{0}\left[\ln \left(1+\frac{1}{x+\eta}\right)-\ln \left(1+\frac{1}{x_{0}+\eta}\right)\right], g(x)=\frac{1}{(x+\eta)(x+\eta+1)} .
$$

For estimates one can put $V_{0} \simeq Z e^{2} / d, \eta \simeq 2 u_{1}^{2} / a_{s}^{2} \equiv \eta_{1}$, where $Z$ is the charge of the nucleus, $u_{1}$ is the amplitude of thermal vibrations, but actually the parameters of potential were determined by means of a fitting procedure using the potential Eq.(2.1) (table of parameters for different crystals is given in Sec.9 of [4]). The function $\kappa(x)$ vanishes at $x=0$ as a result of thermal vibrations and reaches a maximum at

$$
x=x_{m}=\frac{1}{6}\left\{[1+16 \eta(1+\eta)]^{1 / 2}-1-2 \eta\right\}
$$

and then fall off as $x^{-3 / 2}$. It should be noted, that for all crystals $\eta \ll 1$ and in this case $x_{m} \simeq \eta$ and $\kappa\left(x_{m}\right) \equiv \kappa_{m} \simeq \kappa_{s} / \sqrt{\eta}$.

In Fig. 1 the spectral probability of coherent pair creation $d w_{\xi_{+}} / d y$ in tungsten, $\mathrm{T}=293 \mathrm{~K}$, axis $<111>$ is given. The calculation was performed using Eq.(3.7) with term $R_{0}(\lambda)$ only (the corrections $\propto \vartheta_{0}^{2}$ were omitted). The sum of curves at the indicated energy gives unpolarized case. At energies $\omega=100 \mathrm{GeV}$ 
and $\omega=250 \mathrm{GeV}$ the coherent process dominates over the Bethe-Heitler mechanism.

Let us consider the case of small values of parameter $\kappa$. In the constant field approximation (the term with $R_{0}(\lambda)$ in EQ.(3.7) ) in the probability $d W_{\xi_{+}}^{F}\left(\varepsilon_{+}\right)$ one can substitute the asymptotic of McDonald's functions $K_{\nu}(\lambda)$ at $\lambda \gg 1$. After this one obtains using the Laplace method in integration over the transverse coordinate $x$

$$
\frac{d W_{\xi_{+}}^{F}\left(\varepsilon_{+}\right)}{d \varepsilon_{+}}=\frac{\sqrt{3} \alpha m^{2}}{4 \omega^{2} x_{0}}\left[\frac{\varepsilon_{+}}{\omega}\left(1+\xi_{+}\right)+\frac{\varepsilon_{-}^{2}}{\omega^{2}}\right] \frac{\kappa_{m}^{3 / 2}}{\sqrt{-\kappa_{m}^{\prime \prime}}} \exp \left(-\frac{2 \omega^{2}}{3 \varepsilon_{-} \varepsilon_{+} \kappa_{m}}\right)
$$

where $\kappa_{m}=\kappa\left(x_{m}\right), \kappa_{m}^{\prime \prime}=\kappa^{\prime \prime}\left(x_{m}\right)$. For unpolarized particle this result coincides with Eq.(12.14) of [4]. If $\kappa_{m} \ll 1$ one can integrate Eq.(3.11) also using the Laplace method. The integral probability of polarized pair creation is

$$
W_{\xi_{+}}^{F}=\frac{9 \alpha m^{2}}{64 x_{0} \omega} \sqrt{\frac{\pi}{2}} \frac{\kappa_{m}^{2}}{\sqrt{-\kappa_{m}^{\prime \prime}}} \exp \left(-\frac{8}{3 \kappa_{m}}\right)\left(1+\frac{2}{3} \xi_{+}\right) .
$$

At relatively low photon energies where the parameter $\kappa_{m}$ is small

$$
\kappa_{m} \simeq \frac{\kappa_{s}}{\sqrt{\eta}} \simeq \frac{\kappa_{s}}{\sqrt{\eta_{1}}}=\frac{\omega V_{0}}{\sqrt{2} m^{3} u_{1}} \equiv \frac{\kappa_{1}}{\sqrt{2}}
$$

and the main contribution to $e^{+} e^{-}$pair creation gives the incoherent (BetheHeitler) mechanism. The influence of effective crystalline fields on incoherent processes is discussed in [4], Sections 7, 21, but the polarization effects were not included.

In the case where the influence of effective crystalline field is weak $\left(\kappa_{1} \ll 1\right)$, the cross section acquires the correction of the order $\kappa_{1}^{2}$ (we proceed as in Sec.21.4 in [4] but taking into account polarization):

$$
\sigma_{p}\left(\xi_{+}\right)=\frac{1}{2}\left(\sigma_{p}+\xi_{+} \sigma_{\xi_{+}}\right)
$$

where

$$
\begin{aligned}
& \sigma_{p}=\frac{28 Z^{2} \alpha^{3}}{9 m^{2}}\left[\left(1+\frac{396}{1225} \overline{\kappa^{2}}\right) L_{u}-\frac{1}{42}-\frac{1789}{6125} \overline{\kappa^{2}}\right] \\
& \sigma_{\xi_{+}}=\frac{4 Z^{2} \alpha^{3}}{3 m^{2}}\left[\left(1+\frac{32}{75} \overline{\kappa^{2}}\right) L_{u}-\frac{1}{6}-\frac{439}{1125} \overline{\kappa^{2}}\right] \\
& L_{u}=L_{0}-h\left(\frac{u_{1}^{2}}{a^{2}}\right), \quad L_{0}=\ln (m a)+\frac{1}{2}-f(Z \alpha), \quad a=111 Z^{-1 / 3} \lambda(3 .
\end{aligned}
$$

Here $\lambda_{c}$ is the electron Compton wavelength, $\overline{\kappa^{2}}$ is the mean value of $\kappa^{2}(\varrho)$ for the atomic density $n_{a}(\boldsymbol{\varrho})=\exp \left(-\boldsymbol{\varrho}^{2} / 2 u_{1}^{2}\right) / 2 \pi u_{1}^{2}$, the function $h\left(\left(u_{1}^{2} / a^{2}\right)\right.$ reflects a 
nongomogeneity of atomic distribution in crystal, the function $f(Z \alpha)$ represents the Coulomb corrections:

$$
\begin{aligned}
& \overline{\kappa^{2}}=\int \kappa^{2}(\varrho) n_{a}(\varrho) d^{2} \varrho=\int_{0}^{\infty} \frac{e^{-x / \eta_{1}}}{\eta_{1}} \kappa^{2}(x) d x, \eta_{1}=\frac{2 u_{1}^{2}}{a_{s}^{2}} \\
& f(\xi)=\operatorname{Re}[\psi(1+i \xi)-\psi(1)]=\xi^{2} \sum_{n=1}^{\infty} \frac{1}{n\left(n^{2}+\xi^{2}\right)}
\end{aligned}
$$

where $\psi(\xi)$ is the logarithmic derivative of the gamma function, $\operatorname{Ei}(-x)$ is the exponential integral function. It is seen from Eq.(3.15) that in a weak field limit $\left(\kappa_{1} \ll 1\right)$ in the logarithmic approximation the integral positron polarization is $\zeta_{+}=\zeta^{C} \simeq 3 / 7$.

The probability of coherent pair creation becomes comparable with the BetheMaximon probability at $\kappa_{m} \sim 1$. In this situation Eq.(3.12) still has quite satisfactory accuracy (error $<15 \%$ ), while Eq.(3.15) becomes inapplicable. The relative contribution of the LPM effect to the total probability of pair creation by a photon was found recently in 14. This contribution has the maximum about $5 \%$ at $\kappa_{m} \sim 1$ for heavy elements. In this paper we neglect the LPM effect. The differential cross section of incoherent pair creation in the effective crystalline field for polarized particles in the logarithmic approximation for an arbitrary photon energy can be presented in a form

$$
\frac{d \sigma_{p}\left(\xi_{+}\right)}{d y}=\frac{2 Z^{2} \alpha^{3}}{15 m^{2}} L\left(\kappa_{1}\right) \int_{0}^{\infty} \exp \left(-\frac{x}{\eta_{1}}\right) f\left(x, y, \xi_{+}\right) \frac{d x}{\eta_{1}}, \quad L\left(\kappa_{1}\right)=L_{u}+\frac{\ln \left(1+\kappa_{1}\right)}{3}
$$

where

$$
f\left(x, y, \xi_{+}\right)=\left(1+\xi_{+}\right) f_{1}(x, y)+\left[y^{2}\left(1+\xi_{+}\right)+(1-y)^{2}\left(1-\xi_{+}\right)\right] f_{2}(x, y) .
$$

Here

$f_{1}(x, y)=z^{4} \Upsilon(z)-3 z^{2} \Upsilon^{\prime}(z)-z^{3}, \quad f_{2}(x, y)=\left(z^{4}+3 z\right) \Upsilon(z)-5 z^{2} \Upsilon^{\prime}(z)-z^{3}$,

$z=z(x, y)=[\kappa(x) y(1-y)]^{-2 / 3}, \quad y=\frac{\varepsilon_{+}}{\omega}$,

where the function $\kappa(x)$ is defined in Eq.(3.8). Here $\Upsilon(z)$ is the Hardy function

$$
\Upsilon(z)=\int_{0}^{\infty} \sin \left(z \tau+\frac{\tau^{3}}{3}\right)
$$

When the influence of axis field on the incoherent process is weak $\left(\kappa_{1} \ll 1, z \gg\right.$ 1), one can use decomposition (see Appendix D, Eq.(D.3))

$$
\Upsilon(z) \simeq \frac{1}{z}+\frac{2}{z^{4}}+\frac{40}{z^{7}}, \quad f_{1}(z) \simeq 5+\frac{64}{z^{3}}, \quad f_{2}(z) \simeq 10+\frac{86}{z^{3}}
$$


Substituting this decomposition in Eq.(3.17) and integrating over $y$ we obtain within the logarithmic accuracy Eq. (3.15).

Integrating Eq.(3.17) over the positron energy we find for the cross sections contained in Eq. (3.14)

$$
\begin{aligned}
& \sigma_{p}=\frac{4 Z^{2} \alpha^{3}}{15 m^{2}} L_{p}\left(\kappa_{m}\right) \int_{0}^{1} d y \int_{0}^{\infty} \exp \left(-\frac{x}{\eta_{1}}\right)\left[f_{1}(x, y)+2 y^{2} f_{2}(x, y)\right] \frac{d x}{\eta_{1}} \\
& \sigma_{\xi_{+}}=\frac{4 Z^{2} \alpha^{3}}{15 m^{2}} L_{\xi_{+}}\left(\kappa_{m}\right) \int_{0}^{1} d y \int_{0}^{\infty} \exp \left(-\frac{x}{\eta_{1}}\right) f_{1}(x, y) \frac{d x}{\eta_{1}}
\end{aligned}
$$

Here we introduced the functions $L_{p}\left(\kappa_{m}\right)$ and $L_{\xi_{+}}\left(\kappa_{m}\right)$ which refined the logarithmic approximation Eq.(3.17) for $\kappa_{1} \leq 1$ and for $\kappa_{1} \gg 1$. These functions are obtained by means of interpolation procedure ([14):

$$
\begin{aligned}
& L_{p}\left(\kappa_{m}\right)=L_{u}-\frac{1}{42}+\frac{1}{3} \ln \frac{6-3 \kappa_{m}^{2}+3 \kappa_{m}^{3}}{6+\kappa_{m}^{2}} \\
& L_{\xi_{+}}\left(\kappa_{m}\right)=L_{u}-\frac{1}{6}+\frac{1}{3} \ln \frac{9-6 \kappa_{m}^{2}+6 \kappa_{m}^{3}}{9+2 \kappa_{m}^{2}}
\end{aligned}
$$

In Fig.2 the integral probability of pair creation by a photon in tungsten, axis $<111>, T=100 \mathrm{~K}$ as function of photon energy is shown. The curve 1 is the incoherent and the curve 2 is the coherent contribution while the curve 3 is their sum giving the total probability. In low energy region the LPM effect diminishes slightly the total probability (see 14]). In this region the incoherent contribution dominates, these contributions are equal at $\omega \simeq 10 \mathrm{GeV}$. At higher energies the coherent contribution dominates achieving maximum at $\omega \simeq 1.2 \mathrm{TeV}$, while the incoherent contribution aims for zero.

In Fig. 3 the positron polarization vs relative positron energy $\varepsilon / \omega$ is given: the curve 1 for $\omega=12 \mathrm{GeV}$, the curve 2 for $\omega=22 \mathrm{GeV}$, the curve 3 for $\omega=100 \mathrm{GeV}$. Both coherent and incoherent contributions are taken into account. It is seen that shown dependence is approximately universal. When $\varepsilon / \omega \rightarrow 0$ the positron polarization is tending to the Coulomb limit $\zeta^{C}=-1 / 3$ since in this limit the coherent contribution is negligible. In the limit $\varepsilon / \omega \rightarrow 1$ the polarization $\xi_{+} \rightarrow 1$ because of helicity transfer, which is common for both coherent and incoherent contributions.

In Fig.4 the integral positron polarization $\xi_{+}$as a function of photon energy $\omega$ is shown. Both coherent and incoherent contributions are taken into account. At $\omega \rightarrow 0$ this is is the Coulomb limit $\zeta^{C}=3\left(1-1 / 7 L_{u}\right) / 7\left(L_{u}\right.$ is defined in Eq.(3.15)). At intermediate energies the shown dependence is the result of interplay of the coherent and the incoherent contributions, while in the high energy region we see the behavior of the coherent contribution.

Now we turn to the situation when the photon energy is very high and influence of crystalline field becomes very strong $\left(\kappa_{1} \gg 1\right)$. In this case we have (see 
Appendix B) for Eq.(3.14) ( $\sigma_{p}$ is given in Eq.(21.34) in [4])

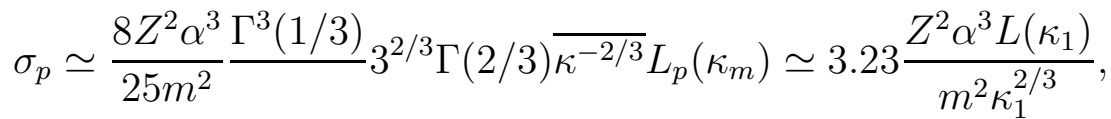

$$
\begin{aligned}
& \sigma_{\xi_{+}} \simeq \frac{1.54 Z^{2} \alpha^{3}}{m^{2}} \overline{\kappa^{-4 / 3}} L_{\xi_{+}}\left(\kappa_{m}\right) \simeq 3.95 \frac{Z^{2} \alpha^{3} L\left(\kappa_{1}\right)}{m^{2} \kappa_{1}^{4 / 3}},
\end{aligned}
$$

where the average $\overline{\kappa^{s}}$ is defined in Eq.(3.16). It should be noted that the asymptotic expansion of $\sigma_{\xi_{+}}$at $\kappa_{1} \gg 1$ becomes valid only for very large values of $\kappa_{1}$ when the ratio $\sigma_{\xi_{+}} / \sigma_{p}$ is very small. This is the consequence of specific form of the next term of decomposition of $\sigma_{\xi_{+}}=\sigma_{\xi_{+}}^{(1)}+\sigma_{\xi_{+}}^{(2)}+\ldots$. The term $\sigma_{\xi_{+}}^{(1)}$ (Eq.(3.24) ) is calculated in Appendix C. The next term $\sigma_{\xi_{+}}^{(2)}$ has a form

$$
\sigma_{\xi_{+}}^{(2)}=\frac{Z^{2} \alpha^{3}}{m^{2}} \frac{A\left(\ln \kappa_{1}+B\right)}{\kappa_{1}^{2}} L_{\xi_{+}}\left(\kappa_{m}\right)
$$

For example, for tungsten crystal, axis $<111>$, $\mathrm{T}=100 \mathrm{~K}$, the obtained in numerical calculation values of the coefficients are $A \simeq 10, B \simeq-1.7$.

In the region $\kappa_{s} \gg 1$ (the constant field approximation is valid if $\kappa_{s}^{1 / 3} \vartheta_{0} / \vartheta_{v} \ll$ 1) the integral probability of coherent pair creation (the terms $\propto \xi_{+}$are calculated in Appendix B , the integral probability for the unpolarized case is given in Eq.(12.16) of [4]) is

$$
\begin{aligned}
& W_{p}^{F}\left(\xi_{+}\right)=c_{0} \frac{\alpha V_{0}}{m x_{0} a_{s} \kappa_{s}^{1 / 3}}\left[\ln \kappa_{s}+B_{1}(\eta)\right. \\
& \left.+\frac{21}{20} \xi_{+}\left(1-\frac{c_{1}}{\kappa_{s}^{2 / 3}}\left(\ln \kappa_{s}+c_{2}\right)\right)\right]
\end{aligned}
$$

where (see Appendix B, Eq.(B.5)

$$
\begin{aligned}
& c_{0}=\frac{5}{7 \pi} \frac{3^{1 / 6} \Gamma^{3}(2 / 3)}{2^{1 / 3} \Gamma(1 / 3)} \simeq 0.201, \quad c_{1}=\frac{2^{7 / 3} \pi}{3^{19 / 6}} \frac{\Gamma(1 / 3)}{\Gamma^{3}(2 / 3)} \simeq 0.527 \\
& c_{2}=\ln 2-\frac{\ln 3}{2}-C+\frac{3}{2} \simeq 1.067 ; \quad \beta=\frac{\eta}{1+\eta} \\
& B_{1}(\eta)=-0.374-3.975 \beta^{2 / 3}\left(1+\frac{8}{15} \beta+\frac{7}{18} \beta^{2}\right)+\beta\left(\frac{3}{2}+\frac{9}{8} \beta+\frac{13}{14} \beta^{2}\right)
\end{aligned}
$$

When $\kappa_{s}=u_{1} \kappa_{1} / a_{s} \gg 1$ the contribution of incoherent process is very small and can be neglected (see Eq.(21.35) of [4]):

$$
\frac{W_{p}^{i n c o h}}{W_{p}^{F}} \leq 10^{-2} \frac{Z \alpha \ln \left(m u_{1} \kappa_{1}^{1 / 3}\right)}{\kappa_{1}^{1 / 3} \ln \kappa_{s}}
$$




\section{Modified theory of coherent pair production}

The estimates of double sum in the phase $A_{p 1}$ made at the beginning of previous section: $\sim\left(\vartheta_{v} / \vartheta_{0}\right)^{2} \Psi$ remain valid also for $\vartheta_{0} \geq \vartheta_{v}$, except that now the factor in the double sum is $\left(\vartheta_{v} / \vartheta_{0}\right)^{2} \leq 1$, so that the values $\left|q_{\|} \tau\right| \geq 1$ contribute. We consider first the limiting case $\vartheta_{0} \gg \vartheta_{v}$, then this factor is small and $\exp \left(i A_{p 1}\right)$ can be expanded accordingly. As a result Eq.(2.9) acquires the form

$$
\begin{aligned}
& d W_{\xi_{+}}^{c o h}(\omega)=\frac{i \alpha m^{2} d \varepsilon_{+}}{4 \pi \omega^{2}} \int_{-\infty}^{\infty} \frac{d \tau}{\tau+i 0} \exp \left(i \frac{m^{2} \omega \tau}{\varepsilon \varepsilon^{\prime}}\right) \sum_{\mathbf{q}, \mathbf{q}^{\prime}} \frac{G(\mathbf{q}) G\left(\mathbf{q}^{\prime}\right)}{m^{2} q_{\|} q_{\|}^{\prime}}\left(\mathbf{q}_{\perp} \mathbf{q}_{\perp}^{\prime}\right) \\
& \times\left[\varphi_{p 2}\left(\xi_{+}\right) \sin \left(q_{\|} \tau\right) \sin \left(q_{\|}^{\prime} \tau\right)+i \varphi_{p 1}\left(\xi_{+}\right) \frac{m^{2} \omega \tau}{\varepsilon_{-} \varepsilon_{+}} \Psi\left(q_{\|}, q_{\|}^{\prime}, \tau\right)\right] \\
& \times \int \frac{d^{3} r}{V} \exp \left[-i\left(\mathbf{q}+\mathbf{q}^{\prime}\right) \mathbf{r}\right] .
\end{aligned}
$$

The integration over coordinate $\mathbf{r}$ in Eq.(4.1) is elementary and gives $\delta_{\mathbf{q}+\mathbf{q}^{\prime}, 0}$, after which the sum over $\mathbf{q}^{\prime}$ and the integrals over $\tau$ are easily calculated by means of the theory of residues. Finally we obtain

$$
\begin{aligned}
& d W_{\xi_{+}}^{c o h}(\omega)=\frac{\alpha d \varepsilon_{+}}{8 \omega^{2}} \sum_{\mathbf{q}}|G(\mathbf{q})|^{2} \frac{\mathbf{q}_{\perp}^{2}}{q_{\|}^{2}}\left[\varphi_{p 2}\left(\xi_{+}\right)+\varphi_{p 1}\left(\xi_{+}\right) \frac{2 m^{2} \omega}{\varepsilon_{-} \varepsilon_{+} q_{\|}^{2}}\left(\left|q_{\|}\right|-\frac{m^{2} \omega}{2 \varepsilon_{-} \varepsilon_{+}}\right)\right] \\
& \times \vartheta\left(\left|q_{\|}\right|-\frac{m^{2} \omega}{2 \varepsilon_{-} \varepsilon_{+}}\right) .
\end{aligned}
$$

For unpolarized photons $\left(\xi_{+}=0\right)$ Eq. (4.2) coincides with the result of standard theory of coherent pair production (CPP), see e.g. [10].

In the case $\kappa_{s} \gg 1\left(\kappa_{s}\right.$ is defined in Eq.(3.8) $)$, one can obtain from general expression Eq.(2.10) the expression for spectral distribution, the region of applicability of which is broader than that of standard CPP theory. For this

purpose it is necessary to take into account that the phase $A_{p 1}$ Eq.(2.10) has for $q_{\|}+q_{\|}^{\prime} \neq 0$ terms of the order $\left(\vartheta_{v} / \vartheta_{0}\right)^{3} / \kappa_{s}^{\prime}$ and $\left(\vartheta_{v} / \vartheta_{0}\right)^{4} / \kappa_{s}^{\prime 2}\left(\kappa_{s}^{\prime}=\kappa_{s} \varepsilon_{-} \varepsilon_{+} / \omega^{2}\right)$ which can be small even for $\vartheta_{0} \leq \vartheta_{v}$ if $\kappa_{s} \gg 1$. Therefore, assuming that these contributions are small, we carry out the corresponding expansion of $\exp \left(i A_{p 1}\right)$, while the term with $q_{\|}+q_{\|}^{\prime}=0$ in the double sum in $A_{p 1}$ will be retained in the exponent. As a result we obtain an expression which coincides in the form with Eq.(4.1) where we must make the substitution

$$
\exp \left(i \frac{m^{2} \omega \tau}{\varepsilon_{-} \varepsilon_{+}}\right) \rightarrow \exp \left(i \frac{m_{*}^{2} \omega \tau}{\varepsilon_{-} \varepsilon_{+}}\right), \quad m_{*}^{2}=m^{2}\left(1+\frac{\varrho}{2}\right) .
$$

Above the parameter $\varrho($ Eq.(2.1) $)$ has the form

$$
\frac{\varrho}{2}=\frac{1}{m^{2}} \sum_{\mathbf{q}, \mathbf{q}^{\prime}} G(\mathbf{q}) G\left(\mathbf{q}^{\prime}\right) \frac{\mathbf{q}_{\perp} \mathbf{q}_{\perp}^{\prime}}{q_{\|} q_{\|}^{\prime}}\left[\delta_{q_{\|}+q_{\|}^{\prime}, 0}-\delta_{q_{\|}, 0} \delta_{q_{\|}^{\prime}, 0}\right]=\sum_{\mathbf{q}, q_{\|} \neq 0} \frac{|G(\mathbf{q})|^{2} \mathbf{q}_{\perp}^{2}}{m^{2} q_{\|}^{2}}
$$


and in the term $\frac{\sin \left(q_{\|}+q_{\|}^{\prime}\right) \tau}{\left(q_{\|}+q_{\|}^{\prime}\right) \tau}$ it is necessary to assume that $q_{\|}+q_{\|}^{\prime} \neq 0$. The remaining calculations are carried out in the same way as in the transition from Eq.(4.1) to Eq.(4.2). The final result can be presented in a form

$$
\begin{aligned}
& d W_{\xi_{+}}^{m c o h}=\frac{\alpha d y}{8 \omega} \sum_{\mathbf{q}}|G(\mathbf{q})|^{2} \frac{\mathbf{q}_{\perp}^{2}}{q_{\|}^{2}}\left[\frac{y}{1-y}\left(1+\xi_{+}\right)+\frac{1-y}{y}\left(1-\xi_{+}\right)\right. \\
& \left.+\frac{8\left(y+\xi_{+}\right)}{(2+\varrho) y} \frac{\tau}{\tau_{0}}\left(1-\frac{\tau}{\tau_{0}}\right)\right] \vartheta\left(\tau_{0}-\tau\right),
\end{aligned}
$$

where

$$
\tau_{0}=\frac{4 \omega\left|q_{\|}\right|}{m^{2}(2+\varrho)}, \quad \tau=\frac{1}{y(1-y)}, \quad \tau_{\min }=4
$$

In derivation of Eq.(4.5) the higher order terms over $\tau \varrho / \tau_{0}(2+\varrho)$ are omitted. Equation (4.5) is not more complicated than Eq.(4.2) but has a significantly broader range of applicability.

The spectral distributions Eqs.(4.2) and (4.5) can be much higher than the Bethe-Heitler pair production distribution for small angles of incidence $\vartheta_{0}$ with respect to selected axis. For the case $\vartheta_{0} \ll 1$ the quantity $q_{\|}$can be represented as

$$
q_{\|} \simeq \frac{2 \pi}{d} m+\mathbf{q}_{\perp} \mathbf{n}_{\perp} .
$$

The main contribution to the sum in Eqs.(4.2) and (4.5) for small $\vartheta_{0}$ is given $\mathbf{q}$ with $m=0$, then

$$
q_{\|} \simeq\left(\frac{2 \pi}{f} k \cos \varphi+\frac{2 \pi}{h} l \sin \varphi\right) \vartheta_{0},
$$

where $f$ and $h$ are the characteristic periods of the potential in the plane transverse to the considered axis, $\varphi$ is the angle of the projection $\mathbf{n}$ onto this plane with respect to one of the planes containing the selected axis, $k$ and $l$ are integers.

Let us consider the spectral distribution of pair production in the extreme limit when the parameter $s=2 \omega\left|q_{\|}\right|_{\min } / m^{2} \sim \omega \vartheta_{0} / m^{2} a_{s} \gg 1$. In this case the maximum of distribution is attained at such values of $\vartheta_{0}$ where the standard CPP becomes inapplicable. Bearing in mind that if $s \gg 1$ and $\vartheta_{0} \sim V_{0} / m$ than $\kappa_{s} \sim s \gg 1$, we can conveniently use the modified theory of CPP. Utilization of the modified theory for these values of $s$ and $\varrho$ gives the exact position of maximum of the spectral distribution (in region where $\tau \sim \tau_{0}$ ) and the value of the total probability within logarithmic accuracy $(\ln s \gg 1)$.

The transverse component of the vector $\mathbf{q}$ in Eq.(4.7) can be selected in a such way that the spectral distribution given by Eq.(4.5) has a sharp maximum near the end of the spectrum at $y_{m}\left(1-y_{m}\right)=(2+\varrho) / 2 s$ with relatively narrow (in terms $1 / s)$ width $\Delta y \sim(1+\varrho / 2) / s$

$$
\left(\frac{d W_{\xi_{+}}}{d y}\right)_{\max }=\frac{\alpha \varrho\left|q_{\|}\right|_{\min }}{4(2+\varrho)}\left(1+\xi_{+}+\frac{1-\xi_{+}}{\tau_{0}^{2}}\right), \quad \tau_{0}=\frac{2 s}{2+\varrho} .
$$


It is seen that the maximum of the spectral distribution with the opposite helicity $\left(\xi_{+}=-1\right)$ is suppressed as $1 / \tau_{0}^{2}$. At $\tau>\tau_{0}$ one have to take into account the next modes of $q_{\|}$(see Eq.(4.7) ). In this part of spectrum the suppression of the probability with opposite helicity is more strong, so the created positrons have nearly complete longitudinal polarization.

Bearing in mind that $\Delta y \sim(2+\varrho) / 2 s$ we find that for $\xi_{+}=1$

$$
\frac{d N_{+}}{d t} \sim \Delta y\left(\frac{d W_{\xi_{+}}}{d y}\right)_{\max } \sim \frac{\alpha \varrho m^{2}}{8 \omega} \sim \frac{\varrho}{L_{\text {rad }}} \frac{\varepsilon_{e}}{\omega},
$$

where $L_{\text {rad }}$ is the radiation length in a corresponding amorphous medium, $\varepsilon_{e}=$ $m\left(16 \pi Z^{2} \alpha^{2} n_{a} \lambda_{c}^{3} L_{0}\right)^{-1}, L_{0}=\ln \left(183 Z^{-1 / 3}-f(Z \alpha)\right)$, the function $\left.f(Z \alpha)\right)$ is defined in Eq.(3.16),$N_{+}$is the number of created positrons, for tungsten one has

$\varepsilon_{e} \simeq 2.5 \mathrm{TeV}$. Thus, the above analysis shows that the considered mechanism of creation of longitudinally polarized positrons is especially effective because there is a gain both in the monochromaticity and the total yield of polarized positrons with the energy $\varepsilon_{+} \simeq \omega$.

\section{Conclusion}

It is shown above that at high energy in the process of production of electronpositron pair with longitudinally polarized particles by the circularly polarized photon in an oriented crystal the phenomenon of helicity transfer takes place in the case when the final particle takes away nearly all energy of the photon. This is true in the constant field limit $\vartheta_{0} \ll V_{0} / m$ as well as in the coherent pair production region $\vartheta_{0}>V_{0} / m$.

In crossing channel: the radiation from longitudinally polarized high energy electrons in oriented crystals is circularly polarized $\left(\xi^{(2)} \rightarrow 1\right)$ near the end of spectrum [12] also in both regions. This is once more the particular case of helicity transfer.

So, the oriented crystal is a very effective device for helicity transfer from a photon to electron or positron and back from an electron to photon. Near the end of spectrum this is nearly $100 \%$ effect.

\section{Acknowledgments}

The authors are indebted to the Russian Foundation for Basic Research supported in part this research by Grant 03-02-16154. 


\section{A Appendix}

\section{Integral polarization of positron in constant field, asymp- totic}

It is instructive that from the expression for $R_{0}(\lambda)$ (Eqs.(3.7), (3.6)) one can find integral degree of positron longitudinal polarization in an external field characterized by parameter $\kappa$ for any $\omega$ : this is ratio of coefficient at $\xi_{+}$and the rest part which enters in the probability for unpolarized particles

$$
\begin{aligned}
& \zeta=\frac{F_{1}}{F}, \quad F_{1}=\int_{0}^{1} \frac{d y}{y}\left[\left(\frac{y}{1-y}-1\right) K_{2 / 3}(\lambda)+\int_{\xi}^{\infty} K_{1 / 3}(z) d z\right], \quad \xi=\frac{2}{3 \kappa y(1-y)}, \\
& F=\int_{0}^{1} d y\left[\left(\frac{y}{1-y}+\frac{1-y}{y}\right) K_{2 / 3}(\lambda)+\int_{\xi}^{\infty} K_{1 / 3}(z) d z\right],
\end{aligned}
$$

where $y=\varepsilon_{+} / \omega$. This result follows also from Eq.(3.70) [4]. The integrand in the first term of $F_{1}$ is antisymmetric at substitution $y \leftrightarrow(1-y)$ and because of this doesn't contributes into integral polarization.

Let us find the asymptotic values of $\zeta$. At $\kappa \ll 1$ it is convenient to write the function $F_{1}$ in the form

$$
F_{1}=\int_{0}^{1} \frac{d y}{y} \int_{a /(4 y(1-y))}^{\infty} K_{1 / 3}(z) d z=\int_{1}^{\infty} \frac{d x}{\sqrt{x(x-1)}} \int_{a x}^{\infty} K_{1 / 3}(z) d z,
$$

where $a=8 / 3 \kappa, x=1 /(4 y(1-y))$. In the limit $a \gg 1$ one can use the standard expansion of $K_{1 / 3}(z)$ for $z \gg 1$ and the last integral in Eq.(A.2) becomes

$$
\int_{a x}^{\infty} K_{1 / 3}(z) d z \simeq \sqrt{\frac{\pi}{2}} \int_{a x}^{\infty} \frac{e^{-z}}{\sqrt{z}} d z \simeq \sqrt{\frac{\pi}{2}} \frac{e^{-a x}}{\sqrt{a x}} .
$$

Taking the integral over $x$ we find

$$
F_{1} \simeq \frac{\pi}{\sqrt{2} a} e^{-a},
$$

while the function $F$ Eq.(A.1) in this terms (see Eq.(3.58) in (4) is

$$
F \simeq \frac{3 \pi}{2 \sqrt{2} a} e^{-a} .
$$

So, we find

$$
\kappa \ll 1, \quad \zeta=\frac{2}{3}
$$


In the limit $\kappa \gg 1$ we present the integral $F_{1}$ as

$$
\begin{aligned}
& F_{1}=\int_{0}^{1} \frac{d y}{y} \int_{a / 4 y(1-y)}^{\infty} K_{1 / 3}(z) d z=F_{1}^{(1)}+F_{1}^{(2)}, \quad a \ll 1, \quad y_{0} \ll 1, \quad \frac{a}{y_{0}} \ll 1 \\
& F_{1}^{(1)}=\int_{y_{0}}^{1} \frac{d y}{y} \int_{a / 4 y(1-y)}^{\infty} K_{1 / 3}(z) d z \simeq \int_{y_{0}}^{1} \frac{d y}{y} \int_{0}^{\infty} K_{1 / 3}(z) d z=\ln \left(\frac{1}{y_{0}}\right) \frac{\pi}{\sqrt{3}} \\
& F_{1}^{(2)}=\int_{0}^{y_{0}} \frac{d y}{y} \int_{a / 4 y(1-y)}^{\infty} K_{1 / 3}(z) d z \simeq \int_{0}^{y_{0}} \frac{d y}{y} \int_{a / 4 y}^{\infty} K_{1 / 3}(z) d z=\int_{a / 4 y_{0}}^{\infty} \frac{d s}{s} \int_{s}^{\infty} K_{1 / 3}(z) d z \\
& \simeq-\ln \left(\frac{a}{4 y_{0}}\right) \frac{\pi}{\sqrt{3}}+\int_{0}^{\infty} \ln s K_{1 / 3}(s) d s \\
& =\frac{\pi}{\sqrt{3}}\left[-\ln \left(\frac{a}{4 y_{0}}\right)+\frac{1}{2}\left(\psi\left(\frac{1}{3}\right)+\psi\left(\frac{2}{3}\right)\right)+\ln 2\right]
\end{aligned}
$$

where $\psi(x)$ is the logarithmic derivative of the gamma function. In calculation of $F_{1}^{(2)}$ we substitute the variable $s=a / 4 y$ and than performed integration by parts. Finally we have for $F_{1}$ in the limit $\kappa \gg 1$

$$
F_{1}=\frac{\pi}{\sqrt{3}}\left(\ln \frac{\kappa}{\sqrt{3}}-C\right) .
$$

Using the two first terms of decomposition of total probability of pair creation (see footnote at p.86 in [4]) we obtain for integral probability of polarized pair creation:

$$
W^{M}\left(\xi_{+}\right)=\frac{\alpha m^{2}}{2 \omega}\left[D \kappa^{2 / 3}-\frac{2}{3}+\frac{\xi_{+}}{3}\left(\ln \frac{\kappa}{\sqrt{3}}-C\right)\right],
$$

where

$$
D=\frac{5 \Gamma(5 / 6)(2 / 3)^{1 / 3}}{14 \Gamma(7 / 6)}=0.37961
$$

So we have for integral degree of longitudinal polarization of created positrons

$$
\zeta(\kappa)=\frac{\ln \kappa-1.126}{1.139 \kappa^{2 / 3}-2}
$$

\section{B Appendix}

\section{Coherent integral polarization term in a crystal at $\kappa_{s} \gg 1$}

Here we consider situation when the value of parameter $\kappa(x)$ on the boundary of cell is small: $\kappa\left(x_{0}\right) \simeq 2 \kappa_{s} / x_{0}^{3 / 2} \ll 1$ and one can extend the integration interval 
over $x$ up to infinity. The term $\propto \xi_{+}$in the integral probability contains the integral (see Appendix A, Eq.(A.2) )

$$
F_{a x}=\int_{0}^{\infty} d x \int_{0}^{1} \frac{d y}{y} \int_{g}^{\infty} K_{1 / 3}(z) d z, \quad a(x)=\frac{8}{3 \kappa(x)} \simeq \frac{4(x+1) \sqrt{x}}{3 \kappa_{s}}
$$

where $g=a(x) /(4 y(1-y))$ The integration interval over $x$ we split into two parts

$$
\text { 1. } x \geq x_{c}, \quad 2 . x \leq x_{c}
$$

where $\kappa_{s}^{3 / 2} \gg x_{c} \gg 1$. In the first interval we have

$$
\begin{aligned}
& F_{a x}^{(1)} \simeq \int_{x_{c}}^{\infty} d x \int_{0}^{1} \frac{d y}{y}\left[\int_{g_{1}(x)}^{\infty} K_{1 / 3}(z) d z-\frac{\sqrt{x}}{3 \kappa_{s} y(1-y)} K_{1 / 3}\left(g_{1}(x)\right)\right] \\
& =\left[\int_{0}^{\infty} d x-\int_{0}^{x_{c}} d x\right] \int_{0}^{1} \frac{d y}{y} \int_{g_{1}(x)}^{\infty} K_{1 / 3}(z) d z-\frac{2}{3} \int_{0}^{1} \frac{d y}{y} \int_{g_{1}\left(x_{c}\right)}^{\infty} K_{1 / 3}(z) d z, \\
& =F_{a x}^{(11)}+F_{a x}^{(12)}+F_{a x}^{(13)}
\end{aligned}
$$

where $g_{1}(x)=x^{3 / 2} /\left(3 \kappa_{s} y(1-y)\right)$. In the first integral over $x: F_{a x}^{(11)}=\int_{0}^{\infty} d x \ldots$ we reverse the integration order, introduce the variable $s=g_{1}(x)$ and integrate over $s$ by parts. We get

$$
F_{a x}^{(11)}=\left(3 \kappa_{s}\right)^{2 / 3} \int_{0}^{1}[y(1-y)]^{2 / 3} \frac{d y}{y} \int_{0}^{\infty} s^{2 / 3} K_{1 / 3}(s) d s=\frac{3^{5 / 3}}{2^{4 / 3}} \frac{\Gamma^{3}(2 / 3)}{\Gamma(1 / 3)} \kappa_{s}^{2 / 3} .
$$

In calculation of integrals over $y$ in the second interval over $x$, as well as in remaining integrals in Eq. (B.2) we use the results of previous Appendix. We have

$$
\begin{aligned}
& F_{a x}^{(2)}+F_{a x}^{(12)}+F_{a x}^{(13)}=-\frac{\pi}{\sqrt{3}}\left[\int_{0}^{x_{c}}\left(\ln \frac{1+x}{x}\right) d x+\frac{2}{3}\left(\ln \frac{2 \kappa_{s}}{\sqrt{3 x_{c}^{3}}}-C\right)\right] \\
& \simeq-\frac{2 \pi}{3 \sqrt{3}}\left(\ln \frac{2 \kappa_{s}}{\sqrt{3}}-C+\frac{3}{2}\right) .
\end{aligned}
$$

Adding Eq.(B.3) and Eq. (B.4) we find

$$
\begin{aligned}
& F_{a x}=c \kappa_{s}^{2 / 3}\left[1-\frac{c_{1}}{\kappa_{s}^{2 / 3}}\left(\ln \kappa_{s}+c_{2}\right)\right], \quad c=\frac{3^{5 / 3}}{2^{4 / 3}} \frac{\Gamma^{3}(2 / 3)}{\Gamma(1 / 3)} \simeq 2.295280 . . \\
& c_{1}=\frac{4 \pi 2^{1 / 3}}{27 \cdot 3^{1 / 6}} \frac{\Gamma(1 / 3)}{\Gamma^{3}(2 / 3)} \simeq 0.526820 . ., \quad c_{2}=\ln 2-\frac{\ln 3}{2}-C+\frac{3}{2} \simeq 1.066625 . .
\end{aligned}
$$




\section{Appendix}

\section{Incoherent integral polarization term in crystal at $\kappa_{1} \gg 1$}

At integration over $y$ the term containing $f_{2}(x, y)$ in Eq. (3.18) vanishes due to symmetry of integrand at $y \leftrightarrow 1-y$ so the integral polarization term (containing $\left.\xi_{+}\right)$is

$$
F_{\text {inc }}=\int_{0}^{\infty} e^{-x / \eta_{1}} \frac{d x}{\eta_{1}} \int_{0}^{1} f_{1}(x, y) d y,
$$

Let us consider the integral

$$
F_{1}=\int_{0}^{1} f_{1}(x, y) d y=2 \int_{0}^{1 / 2} f_{1}(z(x, y)) d y, \quad z=[\kappa y(1-y)]^{-2 / 3},
$$

where $f_{1}(z)$ is defined in Eq.(3.19). Using the differential equation for Hardy function Eq.(D.6) we can represent $f_{1}(z)$ in the form

$$
f_{1}(z)=z^{3} \Upsilon^{\prime \prime}(z)-3 z^{2} \Upsilon^{\prime}(z)
$$

Let us split the integration interval in Eq.(C.2) into two parts

$$
\text { 1. } 0 \leq y \leq y_{0}, \quad 2 . y_{0} \leq y \leq 1 / 2 ; \quad \kappa^{-1} \ll y_{0} \ll 1 .
$$

In the first interval $z \simeq(\kappa y)^{-2 / 3}$ and the corresponding integral is

$$
\begin{aligned}
& F_{1}^{(1)}=\frac{3}{\kappa} \int_{z_{0}}^{\infty}\left(z^{3} \Upsilon^{\prime \prime}(z)-3 z^{2} \Upsilon^{\prime}(z)\right) \frac{d z}{z^{5 / 2}} \\
& =\frac{3}{\kappa}\left[\int_{0}^{\infty} d z-\int_{0}^{z_{0}} d z\right]\left(\sqrt{z} \Upsilon^{\prime \prime}(z)-\frac{3}{\sqrt{z}} \Upsilon^{\prime}(z)\right), \quad z_{0}=\left(\kappa y_{0}\right)^{-2 / 3} \ll 1
\end{aligned}
$$

Now we turn to the first integral

$$
\int_{0}^{\infty}\left(\sqrt{z} \Upsilon^{\prime \prime}(z)-\frac{3}{\sqrt{z}} \Upsilon^{\prime}(z)\right) d z=-\frac{7}{2} \int_{0}^{\infty} \Upsilon^{\prime}(z) \frac{d z}{\sqrt{z}} .
$$

Using the representation Eq. 3.20 we get

$$
\begin{aligned}
& \int_{0}^{\infty} \Upsilon^{\prime}(z) \frac{d z}{\sqrt{z}}=\int_{0}^{\infty} \frac{d z}{\sqrt{z}} \int_{0}^{\infty} \tau \cos \left(z \tau+\frac{\tau^{3}}{3}\right) d \tau=2 \int_{0}^{\infty} d x \int_{0}^{\infty} \tau \cos \left(x^{2} \tau+\frac{\tau^{3}}{3}\right) d \tau \\
& =2 \int_{0}^{\infty} d x \int_{0}^{\infty} \sqrt{\tau} \cos \left(x^{2}+\frac{\tau^{3}}{3}\right) d \tau=\frac{4}{\sqrt{3}} \int_{0}^{\infty} d y \int_{0}^{\infty} d x \cos \left(x^{2}+y^{2}\right) \\
& =\frac{2 \pi}{\sqrt{3}} \int_{0}^{\infty} r \cos \left(r^{2}\right) d r=\frac{\pi}{\sqrt{3}} \int_{0}^{\infty} \cos s d s=0 .
\end{aligned}
$$


The second integral in Eq.(C.4) is

$$
\begin{aligned}
& \int_{0}^{z_{0}}\left(\sqrt{z} \Upsilon^{\prime \prime}(z)-\frac{3}{\sqrt{z}} \Upsilon^{\prime}(z)\right) d z \simeq \int_{0}^{z_{0}}\left(\sqrt{z} \Upsilon^{\prime \prime}(0)-\frac{3}{\sqrt{z}} \Upsilon^{\prime}(0)\right) d z \\
& =\frac{2}{3} z_{0}^{3 / 2}-6 z_{0}^{1 / 2} \Upsilon^{\prime}(0) .
\end{aligned}
$$

In the second interval $y_{0} \leq y \leq 1 / 2$ the variable $z \ll 1$ than the integral in Eq.(C.2) is

$$
F_{1}^{(2)} \simeq-2 \int_{y_{0}}^{1 / 2}\left[\frac{1}{\kappa^{2} y^{2}(1-y)^{2}}+\frac{3 \Upsilon^{\prime}(0)}{\kappa^{4 / 3} y^{4 / 3}(1-y)^{4 / 3}}\right] d y .
$$

In the first term $\left(\propto \kappa^{-2}\right)$ we retain only the main term $\propto 1 / y_{0}$ and in the second term we carry out the integration by parts. We find

$$
F_{1}^{(2)} \simeq-\frac{2}{\kappa^{2} y_{0}}-\frac{18 \Upsilon^{\prime}(0)}{\kappa^{4 / 3} y_{0}^{1 / 3}}+\frac{18 \cdot 2^{5 / 3} \Upsilon^{\prime}(0)}{\kappa^{4 / 3}}-\frac{24 \Upsilon^{\prime}(0)}{\kappa^{4 / 3}} \int_{0}^{1 / 2} \frac{d y}{y^{1 / 3}(1-y)^{7 / 3}} .
$$

Substituting Eq.(C.5)-Eq.(C.7) into Eq.(C.4) and combining the result with Eq.(C.9) we obtain

$$
\begin{aligned}
& F_{1}=F_{1}^{(1)}+F_{1}^{(2)} \simeq \frac{24 \Upsilon^{\prime}(0)}{\kappa^{4 / 3}}\left(3 \cdot 2^{-1 / 3}-b_{1}\right) \\
& b_{1}=\int_{0}^{1 / 2} \frac{d y}{y^{1 / 3}(1-y)^{7 / 3}}=1.86775 . ., \quad F_{1} \simeq \frac{5.7838}{\kappa^{4 / 3}} .
\end{aligned}
$$

Integrating in Eq. (C.1) over $s=x / \eta_{1}\left(\kappa(s) \simeq \kappa_{1} \sqrt{2 s} /(1+s)\right)$ and using the numerical value of the integral

$$
2^{-2 / 3} \int_{0}^{\infty} e^{-s} s^{-2 / 3}(1+s)^{4 / 3}=2.561 . .,
$$

we find

$$
\sigma_{\xi_{+}} \simeq 3.95 \frac{Z^{2} \alpha^{3} L\left(\kappa_{1}\right)}{m^{2} \kappa_{1}^{4 / 3}}
$$

\section{Appendix}

\section{The Hardy function}

The Hardy function

$$
\Upsilon(z)=\int_{0}^{\infty} \sin \left(z \tau+\frac{\tau^{3}}{3}\right) d \tau
$$


is encountered in the theory of electromagnetic processes in an external field.

At $z \ll 1$ the decomposition of $\Upsilon(z)$ is

$\Upsilon(z)=\frac{1}{3^{2 / 3}} \sum_{0}^{\infty} \frac{\left(-3^{1 / 3} z\right)^{k}}{k !} \Gamma\left(\frac{k+1}{3}\right) \cos \left(\frac{k+1}{3} \pi\right)=\frac{\Gamma(1 / 3)}{2 \cdot 3^{2 / 3}}+\frac{\Gamma(2 / 3)}{2 \cdot 3^{1 / 3}} z-\frac{z^{2}}{2}+\ldots$,

and $\Upsilon(0)=0.643950 . ., \Upsilon^{\prime}(0)=0.469447 \ldots$

At $z \gg 1$ there is asymptotic series of $\Upsilon(z)$ over $1 / z^{3}$ :

$$
\Upsilon(z)=\frac{1}{z} \sum_{0}^{\infty} \frac{(3 k) !}{k !} \frac{1}{\left(3 z^{3}\right)^{k}}=\frac{1}{z}\left(1+\frac{2}{z^{3}}+\frac{40}{z^{6}}+\frac{2240}{z^{9}}+\ldots\right)
$$

For calculation it is convenient to use the following representation of the Hardy function and its derivative

$$
\begin{aligned}
& \Upsilon(z)=\int_{0}^{\infty} \sin \left(\frac{\sqrt{3}}{2} z \tau+\frac{\pi}{6}\right) \exp \left(-\frac{z \tau}{2}-\frac{\tau^{3}}{3}\right) d \tau \\
& \Upsilon^{\prime}(z)=\int_{0}^{\infty} \cos \left(\frac{\sqrt{3}}{2} z \tau+\frac{\pi}{6}\right) \exp \left(-\frac{z \tau}{2}-\frac{\tau^{3}}{3}\right) \tau d \tau .
\end{aligned}
$$

It can be obtained using the expression

$$
\Upsilon(z)=\operatorname{Im} \int_{0}^{\infty} \exp \left(i\left(z \tau+\frac{\tau^{3}}{3}\right)\right) d \tau
$$

after a turn of integration line by the angle $\pi / 6$.

The Hardy function satisfies the equation

$$
\Upsilon^{\prime \prime}(z)-z \Upsilon(z)=-1 \text {. }
$$




\section{References}

[1] V. N. Baier, V. M. Katkov, Phys. Lett.,A 25 (1967) 492.

[2] V. N. Baier, V. M. Katkov, Sov.Phys.JETP 26 (1968) 854.

[3] V. N. Baier, V. M. Katkov, Sov.Phys.JETP 28 (1969) 807.

[4] V. N. Baier, V. M. Katkov, V. M. Strakhovenko, Electromagnetic Processes at High Energies in Oriented Single Crystals, World Scientific Publishing Co, Singapore, 1998.

[5] V. B. Berestetskii, E. M. Lifshitz, L. P. Pitaevskii, Quantum Electrodynamics Pergamon Press, Oxford, 1982.

[6] V. N. Baier, V. M. Katkov, V. S. Fadin, Radiation from Relativistic Electrons (in Russian) Atomizdat, Moscow, 1973.

[7] V. N. Baier, V. M. Katkov, V. M. Strakhovenko, Sov.Phys.JETP 63 (1986) 467.

[8] V. N. Baier, V. M. Katkov, V. M. Strakhovenko, Sov.Phys.JETP 65 (1987) 686.

[9] V. N. Baier, A. I. Milstein, V. M. Strakhovenko, Sov.Phys.JETP 42 (1976) 961.

[10] M. L. Ter-Mikaelian, High Energy Electromagnetic Processes in Condensed Media, John Wiley \& Sons, 1972.

[11] V. N. Baier, V. M. Katkov, Phys.Rev. D 62 (2000) 036008.

[12] V. N. Baier, V. M. Katkov, "Radiation from polarized electrons in oriented crystals at high energy" hep-ph/0405046, 2004; Preprint BINP 2004-26, Novosibirsk, 2004, Nucl.Instr.and Meth B, (2005) (in print).

[13] V. N. Baier, V. M. Katkov, "Concept of formation length in radiation theory" hep-ph/0309211, 2003; Physics Reports 409 (2005) 261.

[14] V. N. Baier, V. M. Katkov, "Coherent and incoherent pair creation by a photon in oriented single crystal", hep-ph/0503005, Preprint BINP 2005-7. 


\section{Figure captions}

- Fig.1 The spectral probability of pair creation $d w_{\xi_{+}}^{F} / d y$, the curves 1 and 2 are for energy $\omega=22 \mathrm{GeV}$, the curves 3 and 4 are for energy $\omega=100 \mathrm{GeV}$, the curves 5 and 6 are for energy $\omega=250 \mathrm{GeV}$. The curves 1,3 and 5 are for $\xi=1$, and the curves 2,4 and 6 are for $\xi=-1$.

- Fig.2 The integral probability of pair creation by a photon in tungsten, axis $<111>, T=100 \mathrm{~K}$ as function of photon energy. The curve 1 is incoherent contribution, the curve 2 is coherent contribution the curve 3 is their sum giving the total probability.

- Fig.3 The positron polarization vs relative positron energy $\varepsilon / \omega$ in tungsten, axis $<111>, T=100 \mathrm{~K}$. The curve 1 for $\omega=12 \mathrm{GeV}$, the curve 2 for $\omega=22 \mathrm{GeV}$, the curve 3 for $\omega=100 \mathrm{GeV}$. Both coherent and incoherent contributions are taken into account.

- Fig.4. The integral positron polarization $\xi_{+}$as a function of photon energy $\omega$. 


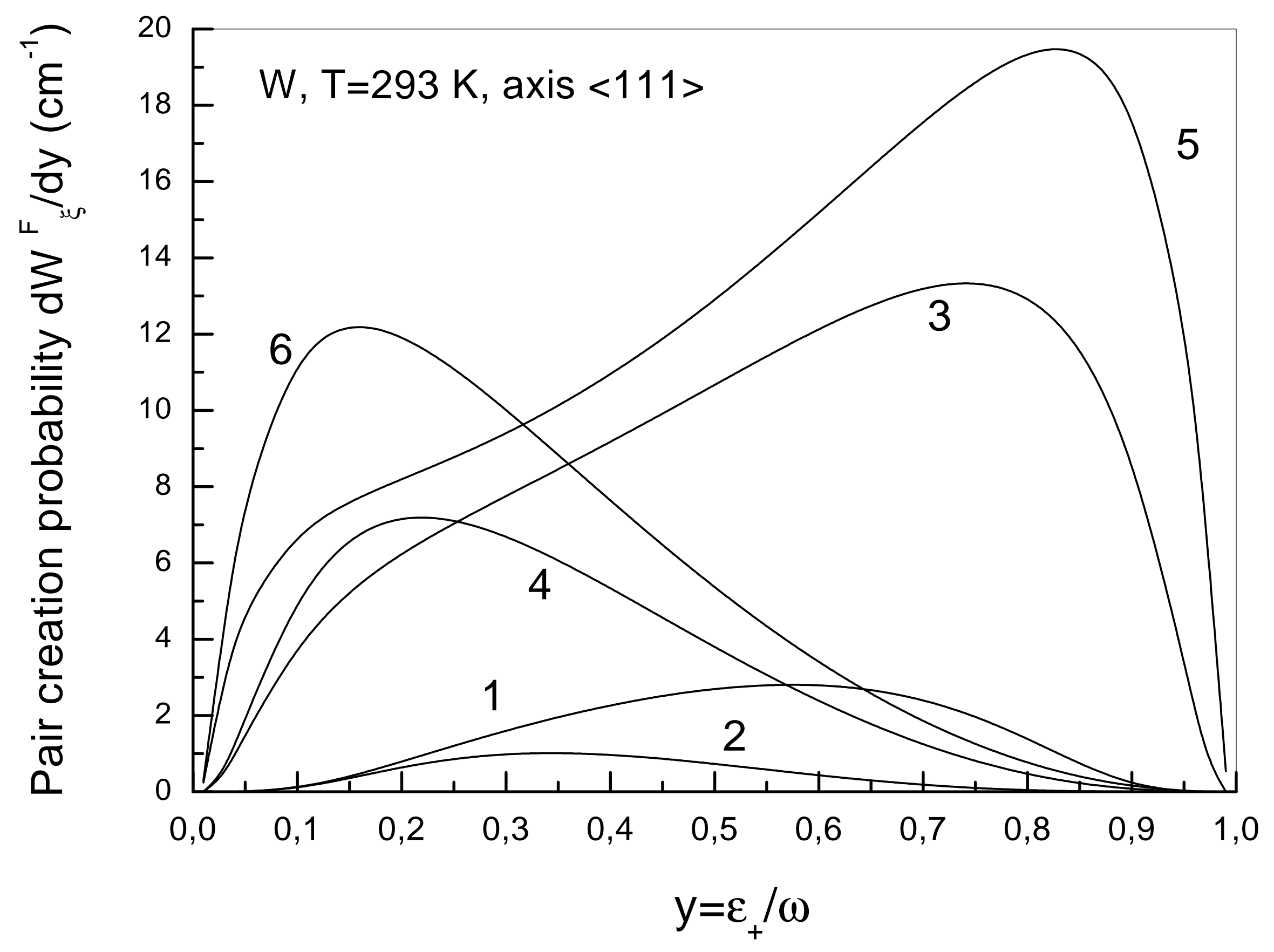




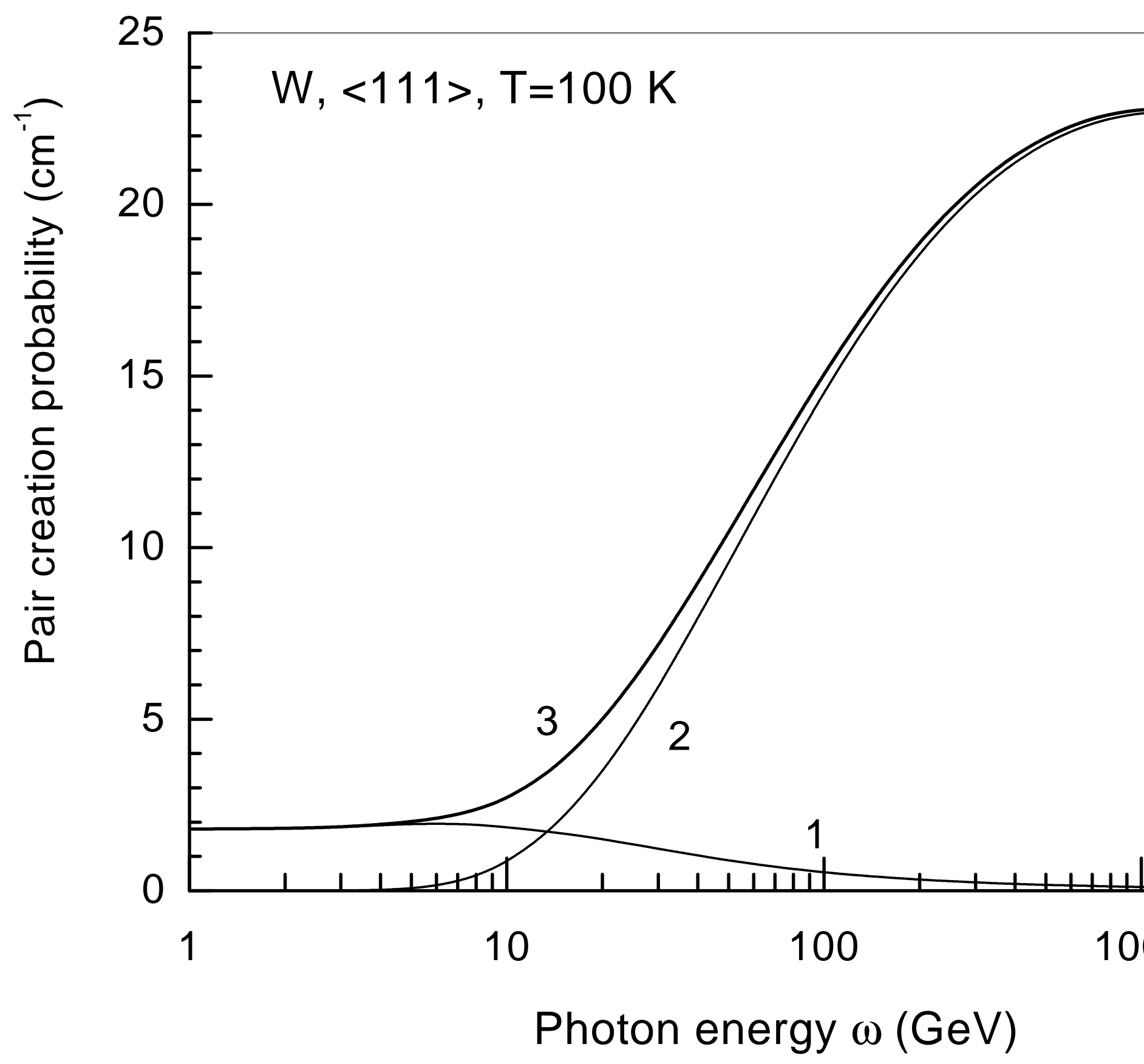




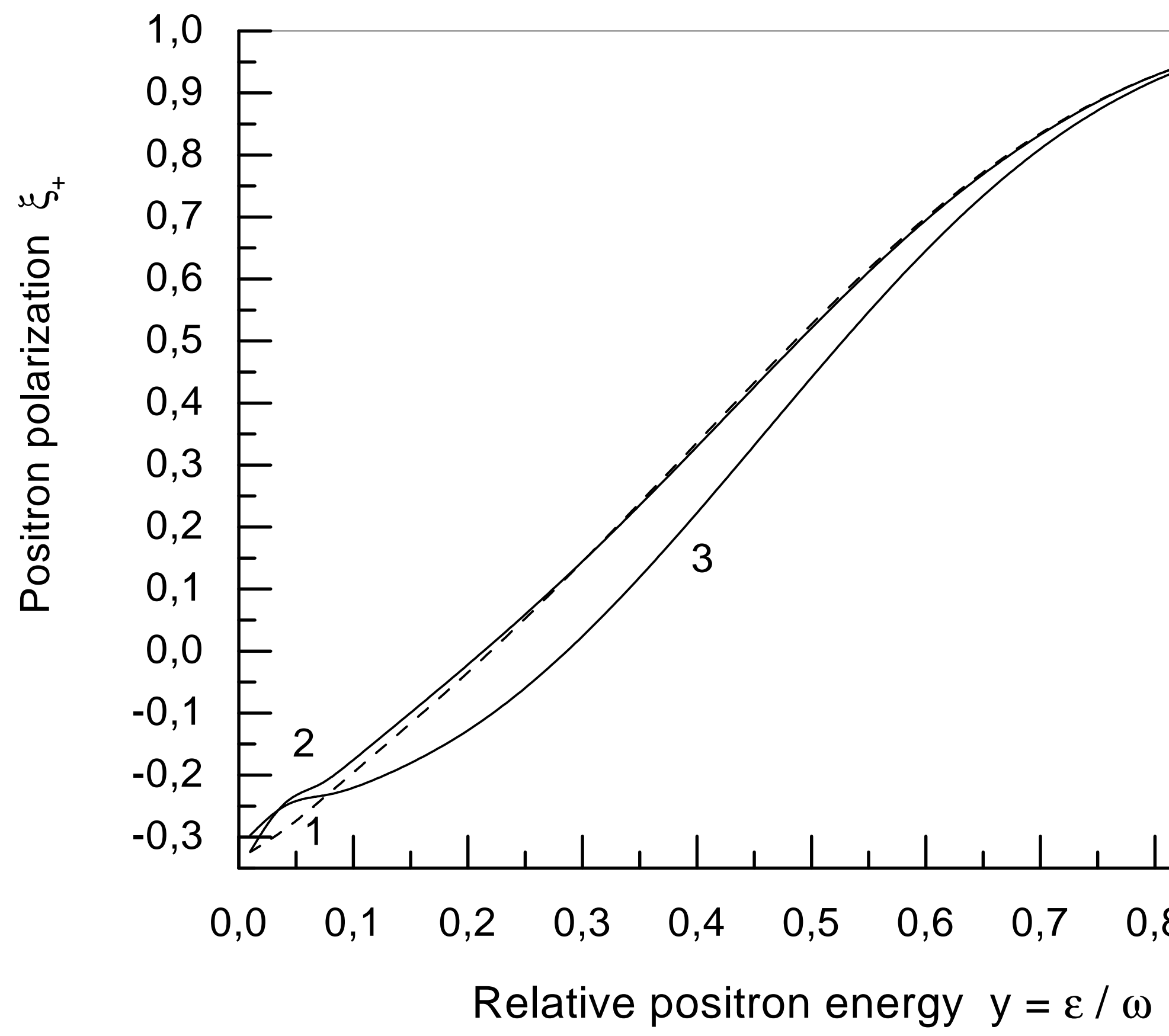




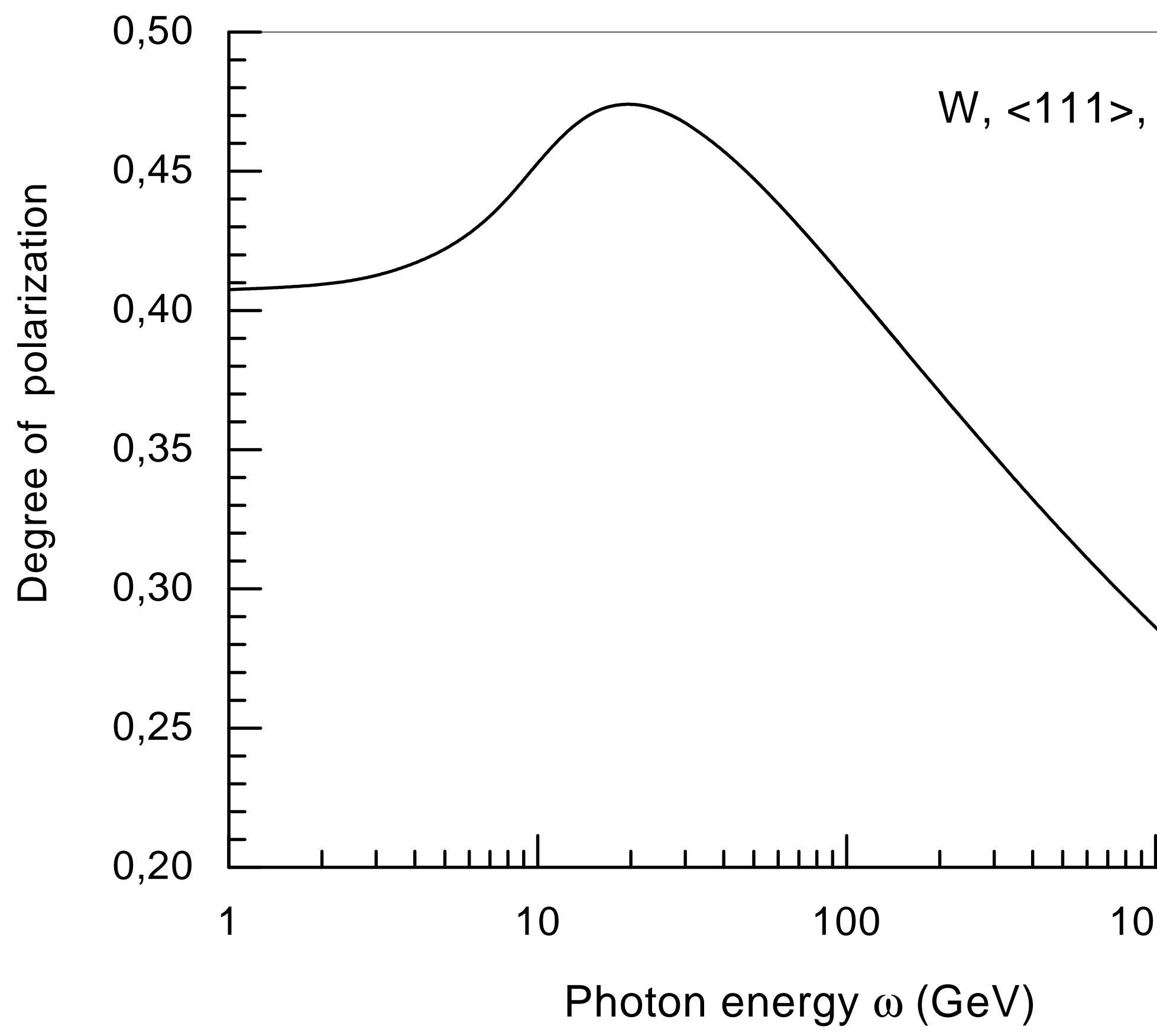

